\title{
Gleiche Wirkung in jedem Klassenzimmer? Moderationseffekte durch motivationale Unterrichtspraktiken am Beispiel einer Nützlichkeitsintervention im Mathematikunterricht und damit einhergehende Herausforderungen
}

\author{
Cora Parrisius, Hanna Gaspard \\ Hector-Institut für Empirische Bildungsforschung, Universität Tübingen, Deutschland \\ Barbara Flunger \\ Universität Utrecht, Niederlande
}

Ulrich Trautwein, Benjamin Nagengast

Hector-Institut für Empirische Bildungsforschung, Universität Tübingen, Deutschland

\section{Dies ist ein Vorabdruck des folgenden Beitrages:}

Parrisius, C., Gaspard, H., Flunger, B., Trautwein, U., \& Nagengast, B., Gleiche Wirkung in jedem Klassenzimmer? Moderationseffekte durch motivationale Unterrichtspraktiken am Beispiel einer Nützlichkeitsintervention im Mathematikunterricht und damit einhergehende Herausforderungen, veröffentlicht in Motivation in unterrichtlichen fachbezogenen LehrLernkontexten, herausgegeben von R. Lazarides \& D. Raufelder, 2021, Springer VS, vervielfältigt mit Genehmigung von Springer Fachmedien Wiesbaden GmbH, ein Teil von Springer Nature. Die finale authentifizierte Version wird in Kürze online verfügbar sein.

\section{Anmerkung}

Die vorliegende Studie wurde mit Mitteln der Deutschen Forschungsgemeinschaft (Projekt Nr. TR 553/7-1) sowie durch das Eliteprogramm für Postdoktorandinnen und Postdoktoranden der Baden-Württemberg Stiftung unterstützt. Cora Parrisius war Doktorandin des LEAD Graduate School \& Research Networks [GSC1028], das durch die Exzellenzinitiative des Bundes und der Länder zur Förderung von Wissenschaft und Forschung an deutschen Hochschulen finanziert wird.

Korrespondenz zu diesem Artikel richten Sie bitte an Cora Parrisius, Hector-Institut für Empirische Bildungsforschung, Universität Tübingen, Walter-Simon-Str. 12, 72072 Tübingen. E-Mail-Adresse: cora.parrisius@uni-tuebingen.de. 


\section{Gleiche Wirkung in jedem Klassenzimmer? Moderationseffekte durch motivationale Unterrichtspraktiken am Beispiel einer Nützlichkeitsintervention im Mathematikunterricht und damit einhergehende Herausforderungen}

\section{Zusammenfassung}

In den vergangenen Jahren wurden zahlreiche Motivationsinterventionen entwickelt und auf ihre Wirksamkeit in der Förderung der Schülerinnen- und Schülermotivation überprüft. Dabei ist eine offene Frage, ob eine Motivationsintervention in Abhängigkeit motivationaler Unterrichtspraktiken im Regelunterricht in jeder Klasse gleichermaßen wirksam ist. Diese Frage bleibt aufgrund mangelnder Power jedoch oftmals unbeantwortet. Anhand einer exemplarischen, auf Klassenebene randomisierten kontrollierten Interventionsstudie mit 82 neunten Klassen ( $n=1916$ ) wurde daher untersucht, (1) welche kleinstmöglichen Moderationseffekte unter gegebenen Parametern mit wünschenswerter Power aufgedeckt werden können und (2) wie viele Klassen für vergleichbare Studien zur Untersuchung von Moderationsfragestellungen mit adäquater Power rekrutiert werden müssten. Zudem wurde untersucht, (3) ob die Effekte der Intervention auf die Wertüberzeugungen und Leistung in Mathematik durch motivationale Unterrichtspraktiken im Regelunterricht moderiert wurden. Die Poweranalysen wiesen auf die Einschränkungen der Studie zur Untersuchung solcher Moderationseffekte hin. Es zeigten sich vereinzelte signifikante Moderationseffekte durch motivationale Unterrichtspraktiken. Diese Ergebnisse und deren Bedeutung für die Planung vergleichbarer Interventionsstudien zur Untersuchung von Moderationseffekten auf Klassenebene werden hinsichtlich der Power und der benötigten Klassenanzahl diskutiert.

Schlüsselwörter: Cluster-Randomized Trial, Intervention, Moderationseffekte, Motivation, Power 


\title{
Same Effect in Every Classroom? Treatment by Moderator Effects of a Relevance Intervention as a Function of Motivational Teaching Practices, and Methodological Challenges
}

\begin{abstract}
Numerous motivation interventions have been developed and tested for their impact on students' motivation. However, it is an open question whether motivation interventions are equally effective in every classroom as a function of motivational teaching practices during regular instruction. This question often remains unanswered due to insufficient power. Based on data from an exemplary cluster-randomized controlled trial with 82 ninth-grade classes $(n$ = 1916), we examined (1) the minimal detectable moderation effect size under given sample parameters with adequate power and (2) which sample size would be necessary for examining moderation effects with adequate power in comparable studies. Additionally, (3) we investigated whether intervention effects on students' math value beliefs and performance were moderated by motivational teaching practices. The power analyses pointed to the limitations of the study to investigate such moderation effects. There were few significant moderation effects through motivational practices during regular instruction. We discuss these results and their significance for designing a comparable cluster-randomized controlled trial in terms of power and the minimum number of classes required.
\end{abstract}

Keywords: cluster-randomized trial, intervention, moderation effects, motivation, power 


\section{Gleiche Wirkung in jedem Klassenzimmer? Moderationseffekte durch motivationale}

\section{Unterrichtspraktiken am Beispiel einer Nützlichkeitsintervention im}

\section{Mathematikunterricht und damit einhergehende Herausforderungen}

Die schulische Motivation von Schülerinnen und Schülern ist ein zentraler Prädiktor für erfolgreiche Lernprozesse (Linnenbrink und Pintrich, 2002). Besorgniserregend ist jedoch, dass die Motivation von Schülerinnen und Schülern im Laufe der Sekundarstufe im Mittel immer weiter absinkt (für einen Überblick siehe Scherrer und Preckel, 2019), insbesondere im Fach Mathematik (z.B. Watt, 2004). In der Vergangenheit konnten erfolgreich verschiedene Interventionen als Ansätze zur Förderung der Motivation im Schulkontext getestet werden, in denen Schülerinnen und Schülern beispielsweise Verknüpfungen des Unterrichtsinhalts mit ihrem eigenen Leben aufgezeigt wurden (Nützlichkeitsinterventionen, bspw. Hulleman und Harackiewicz, 2009; für einen Überblick siehe Lazowski und Hulleman, 2016). Fraglich und für die Praxis relevant ist jedoch, ob solche Interventionen in jedem Kontext gleichermaßen wirksam sind. So ist es beispielsweise denkbar, dass Schülerinnen und Schüler empfänglicher für eine Intervention sind, wenn der in der Intervention vermittelte Inhalt durch einen motivationsförderlichen Unterricht verstärkt wird (bspw. durch eine Lehrkraft, die häufig Verknüpfungen zur Lebenswelt der Schülerinnen und Schüler herstellt; siehe auch Walton und Yeager, 2020). Ebenso ist es denkbar, dass Motivationsinterventionen nur dann wirksam sind, wenn Schülerinnen und Schüler im Regelunterricht eine wenig motivationsförderliche Gestaltung durch die Lehrkraft erfahren und somit ein besonders hoher Bedarf an Motivationsförderung besteht (Rosenzweig und Wigfield, 2016).

Zum Einfluss solcher Kontextmerkmale wie der motivationalen Gestaltung des Regelunterrichts auf die Wirksamkeit einer Motivationsintervention liegen jedoch bislang kaum Forschungsergebnisse vor. Wie wir am Beispiel einer Studie zur Überprüfung einer Nützlichkeitsintervention (MoMa; Gaspard et al., 2015a; Brisson et al., 2017) beleuchten, liegt dies unter anderem an den methodischen Herausforderungen, die mit der Untersuchung 
von Moderationseffekten durch solche Kontextmerkmale einhergehen (siehe auch Spybrook et al., 2016). Trotz ihrer hohen methodischen Qualität (u.a. a priori Power-Analyse zur Untersuchung von Haupteffekten) stößt auch diese exemplarische, auf Klassenebene randomisierte kontrollierte Feldstudie (cluster-randomized trial; CRT) an die Grenzen ihrer Aussagefähigkeit in Bezug auf Moderationseffekte. Beispielhaft für vergleichbare CRTs, in denen also ganze Klassen anstelle von einzelnen Schülerinnen oder Schülern einer der Bedingungen zufällig zugeordnet werden, ermittelten wir mittels post-hoc durchgeführter Poweranalysen, ab welcher Effektstärke Moderationseffekte mit den vorliegenden Stichprobenparametern aufgedeckt werden können und welche Stichprobengröße notwendig gewesen wäre, um tatsächlich zu erwartende Effektgrößen als signifikant anzuzeigen.

Potenzielle Moderationseffekte wurden anschließend mittels Mehrebenenanalysen untersucht. Dabei wurden differenzielle Effekte der MoMa-Intervention auf die Wertüberzeugungen sowie Leistung von Schülerinnen und Schülern der neunten Jahrgangsstufe in Abhängigkeit verschiedener motivationaler Unterrichtspraktiken (Alltagsbezug, Lernunterstützung sowie enthusiastisches Verhalten) in den Blick genommen.

\subsection{Schulische Motivation von Schülerinnen und Schülern}

Die schulische Motivation von Schülerinnen und Schülern umfasst ihre Überzeugungen sowie Werte und Ziele, die zur Wahl einer Aktivität und der Ausdauer in der Verfolgung der Aktivität beitragen (Wentzel und Wigfield, 2009). Genauer sagen laut der Erwartungs-Wert Theorie von Eccles und Kollegen (1983) die Erfolgserwartung („Kann ich es?“) und Wertüberzeugungen (,Was bringt es mir?“) das Lernverhalten, die Leistung sowie akademische Entscheidungen von Schülerinnen und Schülern vorher. Die Wertüberzeugungen bezüglich eines Fachs lassen sich in die wahrgenommene Nützlichkeit, den intrinsischen Wert, die Wichtigkeit und die wahrgenommenen Kosten unterteilen (Eccles, 2005). Die Nützlichkeit gilt dabei im Vergleich zu den restlichen Wertüberzeugungen als am leichtesten von außen beeinflussbar, da sie im Vergleich zu anderen Wertkomponenten weniger in 
individuellen Interessen bzw. der eigenen Identität verankert ist (Harackiewicz et al., 2014). Für die Herausbildung der Wertüberzeugungen von Schülerinnen und Schülern spielt unter anderem das Verhalten der Lehrkraft eine entscheidende Rolle (Eccles, 2007). Entsprechend stehen motivationale Unterrichtspraktiken der Lehrkraft in positivem Zusammenhang mit den Wertüberzeugungen ihrer Schülerinnen und Schüler. Wertüberzeugungen können beispielweise durch Lehrkräfte gefördert werden, indem diese die Relevanz der Inhalte hervorheben (z.B. Rakoczy et al., 2008; Schreier et al., 2014; Schmidt et al., 2019). Auch ein lernunterstützendes Verhalten von Lehrkräften steht in positivem Zusammenhang mit Wertüberzeugungen von Schülerinnen und Schülern (z.B. Wentzel et al., 2010; Dietrich et al., 2015; Lazarides et al., 2019). Je enthusiastischer eine Lehrkraft zudem im Unterricht wahrgenommen wird, desto mehr empfinden Schülerinnen und Schüler selbst Freude im

Unterricht (z.B. Lazarides et al., 2019; siehe auch Keller et al., 2016, für einen Überblick) und berichten zudem positivere Wichtigkeits- und Nützlichkeitswahrnehmungen sowie geringere subjektive Kosten (z.B. Lazarides et al., 2018).

\subsection{Motivationsinterventionen}

Neben dem Unterrichtskontext als Einflussfaktor für die Motivation von Schülerinnen und Schülern sind in den letzten Jahren zahlreiche Motivationsinterventionen als gezielte, in der Regel einmalige und häufig von außen implementierte Maßnahmen zur Förderung der Motivation und der Leistung entwickelt worden (Lazowski und Hulleman, 2016).

Beispielsweise zeigten Interventionen, die die Schülerinnen und Schüler zu Verknüpfungen zwischen dem Unterrichtsinhalt und ihrem Leben anregten, positive Effekte auf ihr Interesse und ihre Leistung (Hulleman und Harackiewicz, 2009; für einen Überblick siehe auch Harackiewicz et al., 2014). Solche Nützlichkeitsinterventionen sind dabei oftmals nicht (nur) im Mittel wirksam in der Förderung verschiedener Motivationskomponenten und der Leistung (bspw. Gaspard et al., 2015a; Brisson et al., 2017), sondern erweisen sich insbesondere als wirksam für bestimmte Risikogruppen (bspw. für Schülerinnen und Schüler mit geringen 
Erfolgserwartungen, Hulleman und Harackiewicz, 2009; oder für Mädchen in Mathematik und Naturwissenschaften, Gaspard et al., 2015a).

Auch die MoMa-Intervention, eine Intervention zur Motivationsförderung im Mathematikunterricht, zielt auf die Förderung der Wertüberzeugungen von Schülerinnen und Schülern der neunten Jahrgangsstufe ab, indem ihnen die Relevanz der Mathematik für verschiedene Bereiche des jetzigen und späteren Lebens mittels einer 90-minütigen Unterrichtseinheit aufgezeigt wird. Die MoMa-Intervention war nicht nur im Mittel wirksam in der Förderung des intrinsischen Werts, Wichtigkeit, Ergebnissen im Leistungstest, Anstrengungsbereitschaft und Erfolgserwartungen (Gaspard et al., 2015a; Brisson et al., 2017), sondern es profitierten Mädchen stärker als Jungen (Gaspard et al., 2015a) sowie diejenigen Schülerinnen und Schüler, deren Eltern ein geringes Mathematikinteresse berichteten (Häfner et al., 2017).

\subsection{Kontextspezifische differenzielle Effekte von Motivationsinterventionen}

Differenzielle Effekte von Motivationsinterventionen wurden in der Vergangenheit vermehrt bezüglich individueller Merkmale von Schülerinnen und Schülern untersucht, die typischerweise mit einer geringeren Motivation in Zusammenhang stehen (bspw. geringeres Vorwissen, geringere Erfolgserwartungen oder geringere motivationale Ressourcen; für einen Überblick siehe bspw. Rosenzweig und Wigfield, 2016). Es ist jedoch ebenso vorstellbar, dass eine unterschiedliche Wirksamkeit einer Intervention für ganze Klassen vorliegt. Schülerinnen und Schüler erleben unterschiedlich motivationale Lernumgebungen in ihrem Schulalltag. Diese Unterschiedlichkeit in den motivationalen Unterrichtspraktiken im Regelunterricht kann nicht nur in direktem Zusammenhang mit der Motivation (z.B. Dietrich et al., 2015; Lazarides et al., 2019; Schmidt et al., 2019) und dem Engagement (z.B. Reeve et al., 2004; Wentzel, 2009; Frenzel et al., 2010; Zhang, 2013) der Schülerinnen und Schüler stehen, sondern gleichermaßen den Erfolg einer Nützlichkeitsintervention befördern (Potenzialperspektive) oder beeinträchtigen (defizitäre Perspektive). Walton und Yeager 
(2020) argumentieren, dass eine Intervention nur dann wirksam sein kann, wenn sie auf einen unterstützenden Kontext trifft, da nur ein kongruenter Kontext die Akzeptanz, Übernahme und Fortdauer der Interventionsbotschaft durch die Schülerinnen und Schüler ermögliche. Ebenso ist denkbar, dass eine Intervention nur in einem wenig motivationsförderlichen Kontext ihre gesamte Wirkkraft entfalten kann, da ein höherer Bedarf einer solchen Intervention vorliegt (Rosenzweig und Wigfield, 2016).

Einige Studien weisen bereits darauf hin, dass sowohl formale als auch informale Kontextressourcen den Erfolg von Interventionen beeinflussen können (bspw. in Abhängigkeit der Leistungen und Einstellungen der Mitschülerinnen und -schüler; Yeager et al., 2019; Walton und Brady, 2020). Darüber hinaus ist jedoch die Frage weitestgehend unbeantwortet, ob der Erfolg einer Nützlichkeitsintervention vom motivationalen Unterrichtskontext, in dem eine Intervention implementiert wird und der eigenständig bereits ähnliche Effekte wie die Intervention aufweist, abhängt. Für die Weiterentwicklung und potenzielle flächendeckende Implementation von Motivationsinterventionen ist es wichtig, solche Kontextmerkmale und ihre Auswirkungen auf eine erfolgreiche Implementation zu untersuchen (Yeager et al., 2019). Allerdings ist dies bei weitem keine gängige Praxis vielmehr findet sich unseres Wissens nach keine Untersuchung, die motivationale Unterrichtspraktiken der Lehrkraft als Moderatoren berücksichtigt.

\subsection{Methodische Herausforderungen bei der Aufdeckung von Moderationseffekten}

Dass Moderationseffekte in Abhängigkeit von Kontextmerkmalen so selten in den Blick genommen werden, lässt sich unter anderem auf methodische Herausforderungen bei der Untersuchung von Moderationseffekten zurückführen. Bei der Aufdeckung von tatsächlich vorhandenen oder „,wahren“ Effekten der Intervention in einem CRT spielt insbesondere die Teststärke (oder auch gängiger der englische Begriff Power) einer Studie eine entscheidende Rolle (Snijders, 2005). Ist die Power zur Aufdeckung von Moderationseffekten zu gering, werden wahre Moderationseffekte mit erhöhter 
Wahrscheinlichkeit als nicht signifikant angezeigt und folglich übersehen ( $\beta$-Fehler). Zugleich überschätzen statistisch signifikante Moderationseffekte in der Stichprobe den wahren Moderationseffekt deutlich (Gelman und Carlin, 2014).

Um eine adäquate Power (bspw. 0,80; siehe z.B. Muthén und Muthén, 2002) zur Aufdeckung von Interventionseffekten zu erreichen, ist insbesondere die Rekrutierung einer angemessenen Anzahl an Klassen entscheidend (Raudenbush und Liu, 2000). Bei identischer Stichprobengröße und unter der Annahme vergleichbar hoher Regressionskoeffizienten für Haupt- und Moderationseffekte ist die Power zur Aufdeckung eines Moderationseffekts jedoch um ein Vielfaches kleiner als jene zur Aufdeckung eines Haupteffekts (Spybrook et al., 2016). Um dieselbe Power zur Untersuchung von Moderationseffekten zu erreichen, ist teilweise eine vielfach größere Stichprobe nötig.

CRTs werden neben der Untersuchung von Haupteffekten einer Intervention oftmals auch für die Untersuchung von Moderationseffekten genutzt - auch wenn dies regelmäßig nicht vorab geplant und die Studie entsprechend angelegt wurde. Folglich ist zum einen interessant, wie informativ Moderationsanalysen mit gegebenen Datenkonstellationen überhaupt sein können (d.h. welche Effektgrößen aufgedeckt werden könnten). Zeitgleich ist es aber auch wichtig zu wissen, in welcher Größenordnung zukünftige CRTs angelegt sein müssten, um eine adäquate Power zur Untersuchung von Moderationseffekten zu erzielen. Mittels der Untersuchung eines exemplarischen CRTs (MoMa) liefert dieser Beitrag daher Einblicke in die kleinstmögliche Effektgröße, die mit einer vergleichbaren Interventionsstudie mit adäquater Power aufgedeckt werden kann, und in die Anzahl der Klassen, die zur Beantwortung von zukünftigen Moderationsfragestellungen unter ähnlichen Bedingungen rekrutiert werden müsste.

\subsection{Ziele der vorliegenden Studie}

Nützlichkeitsinterventionen können die Leistung und die Motivation von Schülerinnen und Schülern fördern (Lazowski und Hulleman, 2016). Unklar ist bislang jedoch 
weitestgehend, inwiefern der Unterrichtskontext (bspw. motivationale Unterrichtspraktiken im Regelunterricht) die Wirksamkeit einer Nützlichkeitsintervention beeinflussen kann. Mittels einer beispielhaften Nützlichkeitsinterventionsstudie (der MoMa-Studie) wollen wir daher der Frage nachgehen, inwiefern die alltagsbezogene, lernunterstützende und enthusiastische Gestaltung des Regelunterrichts durch die Lehrkraft den Erfolg der MoMaIntervention beeinflusst. Die MoMa-Studie wird hierbei stellvertretend als Beispiel einer auf Klassenebene randomisierten kontrollierten Feldstudie untersucht. Sie wurde auf Grundlage einer vorab für die Haupteffekte durchgeführten Poweranalyse geplant (mit einer Power von 0,73, um Haupteffekte von 0,20 aufzudecken; Gaspard et al., 2015a). Nichtsdestotrotz stößt auch die MoMa-Studie in Bezug auf Moderationseffekte an die Grenzen ihrer statistischen Aussagekraft.

In diesem Artikel möchten wir daher die folgenden Fragen beantworten: (1) Welches sind die kleinstmöglichen Moderationseffekte, die mit einer auf Klassenebene randomisierten kontrollierten Feldstudie (wie bspw. der MoMa-Studie) aufgedeckt werden können? (2) Welche Stichprobengröße wäre für Studien zur Aufdeckung typischerweise erwartbarer Moderationseffekte mit akzeptabler Power notwendig? (3) Finden sich differenzielle Effekte der MoMa-Intervention auf die Wertüberzeugungen und Leistung in Abhängigkeit verschiedener motivationaler Unterrichtspraktiken (Alltagsbezug, Lernunterstützung und enthusiastisches Verhalten)?

\section{Methode}

Eine Zustimmung zur Durchführung dieser Studie wurde vorab durch die Ethikkommission der Wirtschafts- und Sozialwissenschaftlichen Fakultät der Universität Tübingen erteilt.

\subsection{Stichprobe und Prozedere}

Zur Untersuchung der Forschungsfragen wurden Reanalysen der MoMa-Studie aus dem Schuljahr 2012/13 (Gaspard et al., 2015a; Brisson et al., 2017) durchgeführt. An dieser 
Studie nahmen 1978 Schülerinnen und Schüler der neunten Jahrgangsstufe aus 82 Klassen von 25 baden-württembergischen Gymnasien nach aktivem Elterneinverständnis teil. Da 62 Schülerinnen und Schüler am Tag der Intervention abwesend waren, wurden sie konsistent zu vorherigen Untersuchungen (Gaspard et al., 2015a; Brisson et al., 2017) ausgeschlossen. Die Analysen basieren folglich auf einer Stichprobe von 1916 Schülerinnen und Schülern (53,5\% weiblich; Alter zu Beginn der Studie $M=14.7$ Jahre, $S D=0.6$ ). Die Mathematiklehrkräfte und ihre Klassen wurden randomisiert der Textbedingung (30 Klassen), der Zitatebedingung (25 Klassen) oder der Wartekontrollbedingung (27 Klassen) zugewiesen.

Die 90-minütige Intervention wurde durch fünf Doktorandinnen implementiert. Die Schülerinnen und Schüler wurden zu Beginn des Schuljahres (Oktober 2012; Prätest), durchschnittlich 6 Wochen (Posttest), als auch durchschnittlich 5 Monate nach der Intervention (Follow-Up) zu ihren Wertüberzeugungen sowie zum Prätest zu ihrer Wahrnehmung motivationaler Unterrichtspraktiken im Regelunterricht befragt und absolvierten zum Follow-Up einen normierten Leistungstest.

\subsection{Nützlichkeitsintervention}

Die MoMa-Intervention war in den Mathematikunterricht eingebettet und sollte den Nutzen der Mathematik aufzeigen (Gaspard et al., 2015a; Brisson et al., 2017). Zunächst verfolgten die Schülerinnen und Schüler eine zweiteilige psychoedukative Präsentation. Im ersten Teil wurden ihnen Forschungsergebnisse zur Bedeutung von Anstrengungsbereitschaft und Selbstkonzept für die Leistung in Mathematik sowie zu den Effekten unterschiedlicher Vergleichsprozesse im Klassenzimmer vorgestellt. Der zweite Teil enthielt Beispiele zur Relevanz der Mathematik für verschiedene Studiengänge und Ausbildungen, Berufsmöglichkeiten sowie Freizeitaktivitäten. Die Präsentation unterschied sich nicht zwischen den beiden Interventionsbedingungen.

In der zweiten Hälfte der 90-minütigen Intervention bearbeiteten die Schülerinnen und Schüler eigenständig einen Arbeitsauftrag, der sich je nach Bedingung unterschied. In der 
Textbedingung erhielten die Schülerinnen und Schüler die Aufgabe, Argumente für den persönlichen Nutzen von Mathematik zu sammeln und schriftlich zu erläutern. Schülerinnen und Schüler der Zitatebedingung erhielten sechs verschriftlichte Interviewzitate junger Erwachsener über den Nutzen von Mathematik, die sie in Bezug auf ihre persönliche Relevanz bewerten sollten. Schülerinnen und Schüler der Wartekontrollbedingung erhielten nach Abschluss der Datenerhebungen die erfolgreichere der beiden Interventionen (d.h. das Bewerten von Zitaten; für mehr Informationen siehe Gaspard et al., 2015a; Brisson et al., 2017).

\subsection{Instrumente}

Die Wertüberzeugungen und motivationalen Unterrichtspraktiken wurden anhand einer vierstufigen Ratingskala (von 1 ,stimmt gar nicht“ bis 4 ,stimmt genau“) eingeschätzt. Beispielitems, Reliabilitäten sowie Korrelationen zwischen den Skalen finden sich in Tab. 1 und 2.

\section{Tabelle 1}

Beispielitems und Reliabilitäten der verwendeten Skalen zu allen Messzeitpunkten

\begin{tabular}{llccc}
\hline \multicolumn{1}{c}{ Variable } & \multicolumn{1}{c}{ Beispielitem } & $\alpha_{\mathrm{T} 1}$ & $\alpha_{\mathrm{T} 2}$ & $\alpha_{\mathrm{T} 3}$ \\
\hline Nützlichkeit & $\begin{array}{l}\text { Ich werde die Mathematik in meinem Leben } \\
\text { noch oft benötigen. }\end{array}$ & 0,84 & 0,86 & 0,87 \\
Intrinsischer & Mathematik macht mir Spaß. & 0,94 & 0,93 & 0,92 \\
Wert & Mathematik ist mir persönlich sehr wichtig. & 0,91 & 0,92 & 0,92 \\
Wichtigkeit & $\begin{array}{l}\text { Um in Mathematik gut zu sein, müsste ich viel } \\
\text { Kosten }\end{array}$ & 0,93 & 0,94 & 0,94 \\
& Freizeit opfern. & & & \\
\hline
\end{tabular}

Anmerkung. Eine vollständige Liste der Items kann in Gaspard et al. (2015b) eingesehen werden.

2.3.1. Wertüberzeugungen. Die Wertüberzeugungen der Schülerinnen und Schüler wurden basierend auf der EWT (Eccles et al., 1983) in Bezug auf die Dimensionen Nützlichkeit (12 Items), intrinsischer Wert (4 Items), Wichtigkeit (10 Items) und Kosten (11 
Items) zu allen drei Testzeitpunkten erfasst. Konfirmatorische Faktorenanalysen bestätigten die Trennbarkeit dieser Dimensionen (Gaspard et al., 2015b).

2.3.2. Mathematik-Leistung. Als Prätest-Messung für die Leistung der Schülerinnen und Schüler dienten die Ergebnisse der landesweiten Lernstandserhebungen in BadenWürttemberg zu Beginn von Klassenstufe 9. Sie umfassten 38 Aufgaben zu den Themenbereichen Algebra, Geometrie sowie Wahrscheinlichkeitsrechnung, wobei die Leistung in Prozent korrekt gelöster Aufgaben angegeben wurde. Zum Follow-Up wurde die Leistung der Schülerinnen und Schüler mittels eines 3-minütigen normierten Speed-Tests gemessen, der 50 Aufgaben zum Umgang mit typischen mathematischen Operationen umfasste (maximal erreichbare Punktzahl = 50; Schmidt et al., 2013).

Tabelle 2

Interkorrelationen der Wertüberzeugungen über alle Messzeitpunkte

\begin{tabular}{|c|c|c|c|c|c|c|c|c|c|c|c|c|c|}
\hline & & (1) & (2) & (3) & (4) & (5) & (6) & (7) & (8) & (9) & (10) & $(11)$ & (12) \\
\hline (1) & Nützlichkeit T1 & - & & & & & & & & & & & \\
\hline (2) & Nützlichkeit T2 & 0,69 & - & & & & & & & & & & \\
\hline (3) & Nützlichkeit T3 & 0,62 & 0,69 & - & & & & & & & & & \\
\hline (4) & Intrins. Wert T1 & 0,54 & 0,41 & 0,38 & - & & & & & & & & \\
\hline (5) & Intrins. Wert T2 & 0,46 & 0,51 & 0,42 & 0,79 & - & & & & & & & \\
\hline (6) & Intrins. Wert T3 & 0,42 & 0,42 & 0,51 & 0,74 & 0,79 & - & & & & & & \\
\hline (7) & Wichtigkeit T1 & 0,68 & 0,54 & 0,46 & 0,64 & 0,54 & 0,49 & - & & & & & \\
\hline (8) & Wichtigkeit T2 & 0,56 & 0,67 & 0,53 & 0,52 & 0,60 & 0,52 & 0,76 & - & & & & \\
\hline (9) & Wichtigkeit T3 & 0,50 & 0,54 & 0,66 & 0,47 & 0,50 & 0,59 & 0,68 & 0,76 & - & & & \\
\hline (10) & Kosten T1 & $-0,31$ & $-0,24$ & $-0,24$ & $-0,68$ & $-0,56$ & $-0,55$ & $-0,41$ & $-0,34$ & $-0,33$ & - & & \\
\hline (11) & Kosten T2 & $-0,30$ & $-0,26$ & $-0,27$ & $-0,61$ & $-0,61$ & $-0,58$ & $-0,38$ & $-0,39$ & $-0,36$ & 0,78 & - & \\
\hline (12) & Kosten T3 & $-0,27$ & $-0,24$ & $-0,24$ & $-0,56$ & $-0,54$ & $-0,59$ & $-0,35$ & $-0,34$ & $-0,36$ & 0,73 & 0,82 & - \\
\hline
\end{tabular}

Anmerkung. Alle Korrelationen sind signifikant mit $p<0,001$.

2.3.3. Motivationale Unterrichtspraktiken. Zur Erfassung motivationaler Unterrichtspraktiken der Lehrkraft im Regelunterricht zum Prätest berichteten die Schülerinnen und Schüler den wahrgenommenen Alltagsbezug (3 Items; bspw. Baumert et al., 2009) sowie die wahrgenommene Lernunterstützung durch die Lehrkraft (7 Items; Lazarides et al., 2019; siehe auch Baumert et al., 2009). Darüber hinaus berichteten sie ihre 
Wahrnehmung des enthusiastischen Verhaltens der Lehrkraft im Mathematikunterricht (3 Items; Baumert et al., 2009).

\subsection{Statistisches Vorgehen}

2.4.1. Poweranalysen. In einem ersten Schritt interessierte uns die (1) minimal aufdeckbare Effektgröße im Vergleich zum Haupteffekt (minimum detectable effect size difference, MDESD) eines CRTs wie der MoMa-Studie und die (2) minimal erforderliche Stichprobengröße (minimum required sample size, MRSS), um eine adäquate Power zu erreichen. Hierfür führten wir pro interessierendem Parameter (MDESD, MRSS), Outcome (Nützlichkeit, Wichtigkeit, intrinsischer Wert, Kosten, Leistung) und Messzeitpunkt (Posttest, Follow-Up) je eine Poweranalyse mit dem R-Package PowerUpR v1.0.3 (Bulus et al., 2019) durch. Da in einer Poweranalyse jeweils nur eine Interventionsbedingung im Verhältnis zur Kontrollbedingung berücksichtigt werden kann, wurden diese Analysen zusätzlich getrennt für Text- und Zitatebedingung durchgeführt. Dabei lagen den Analysen eine angenommene Power von 0,80 und für die MRSS ein zu erwartender Moderationseffekt von 0,10 (bzw. 0,15) zugrunde. Die zu erwartenden Effektgrößen lehnen sich zum einen an Werte von Spybrook und Kollegen an (2016) und wurden zum anderen an die gefundenen Haupteffekte (zwischen 0,12 und 0,30; Gaspard et al., 2015a; Brisson et al., 2017) angelehnt unter Berücksichtigung, dass Moderationseffekte typischerweise geringer als Haupteffekte ausfallen (Aguinis et al., 2005).

Die für die Bestimmung der MDESD und MRSS relevanten Strichprobenparameter Intraklassenkorrelation (ICC) der interessierenden Outcome-Variablen, Anteil der erklärten Varianz durch den Prätestwert auf Individualebene $\left(R_{1}^{2}\right)$ und Klassenebene $\left(R_{2}^{2}\right)$, das Verhältnis von Schülerinnen und Schülern der Interventionsbedingung zur Wartekontrollbedingung (P), durchschnittliche Anzahl der Schülerinnen und Schüler pro Klasse ( $n$ ) und Anzahl der Klassen pro Bedingung $(J)$ - wurden post-hoc aus den Daten der MoMa-Studie ermittelt. Die ICC sowie $R_{1}^{2}$ und $R_{2}^{2}$ wurden dabei analog zum Vorgehen nach 
Dong und Kollegen (2016) separat pro Outcome und Testzeitpunkt ermittelt (siehe Tab. 3).

Die Substichproben bestehend aus Schülerinnen und Schülern der Textbedingung/der

Zitatebedingung je inklusive der Wartekontrollbedingung wiesen ein Verhältnis von $\mathrm{P}_{\mathrm{Text}}=$

$0,53 / \mathrm{P}_{\text {Zitate }}=0,48$ auf. Es wurde jeweils $\mathrm{g}^{*}=1$ weitere Kovariate auf Klassenebene

berücksichtigt (der Prätestwert), in einer Klasse waren durchschnittlich $n_{\text {Text }}=24 / n_{\text {Zitate }}=23$

Schülerinnen und Schüler und die Substichproben umfassten $J_{\text {Text }}=57 / J_{\text {Zitate }}=52$ Klassen.

\section{Tabelle 3}

Stichprobenparameter je nach Interventionsbedingung (jeweils inkl. Wartekontrollbedingung) als Grundlage für die Durchführung der Poweranalysen

\begin{tabular}{|c|c|c|c|c|c|c|c|c|c|}
\hline & \multicolumn{2}{|c|}{ Nützlichkeit } & \multicolumn{2}{|c|}{ Intrins. Wert } & \multicolumn{2}{|c|}{ Wichtigkeit } & \multicolumn{2}{|c|}{ Kosten } & \multirow{2}{*}{$\begin{array}{c}\text { Leistung } \\
\mathrm{T} 3\end{array}$} \\
\hline & $\mathrm{T} 2$ & T3 & $\mathrm{T} 2$ & T3 & $\mathrm{T} 2$ & T3 & $\mathrm{T} 2$ & $\mathrm{~T} 3$ & \\
\hline \multicolumn{10}{|c|}{ Textbedingung } \\
\hline ICC & 0,08 & 0,09 & 0,08 & 0,07 & 0,05 & 0,07 & 0,05 & 0,06 & 0,03 \\
\hline$R_{1}^{2}$ & 0,43 & 0,35 & 0,61 & 0,53 & 0,56 & 0,43 & 0,59 & 0,50 & 0,27 \\
\hline$R_{2}^{2}$ & 0,76 & 0,83 & 0,77 & 0,76 & 0,63 & 0,58 & 0,73 & 0,65 & 0,34 \\
\hline \multicolumn{10}{|c|}{ Zitatebedingung } \\
\hline ICC & 0,06 & 0,04 & 0,04 & 0,04 & 0,03 & 0,03 & 0,03 & 0,04 & 0,02 \\
\hline$R_{1}^{2}$ & 0,50 & 0,40 & 0,63 & 0,54 & 0,58 & 0,51 & 0,60 & 0,55 & 0,24 \\
\hline$R_{2}^{2}$ & 0,63 & 0,67 & 0,50 & 0,54 & 0,55 & 0,73 & 0,39 & 0,30 & 0,04 \\
\hline
\end{tabular}

Anmerkung. $\mathrm{T} 2=$ Posttest; T3 = Follow-Up. ICC = Intraklassenkorrelationskoeffizient; $R_{1}^{2}=$ Anteil der erklärten Varianz auf Individualebene durch Prätestwert; $R_{2}^{2}=$ Anteil der erklärten Varianz auf Klassenebene durch Prätestwert.

2.4.2. Moderationsanalysen. Zur Beantwortung der Frage nach differenziellen

Effekten der MoMa-Intervention in Abhängigkeit verschiedener motivationaler

Unterrichtspraktiken führten wir Mehrebenenregressionsanalysen in Mplus 7.31 (Muthén und

Muthén, 1998-2015) durch. Dabei orientierten sich die Modelle an Gaspard et al. (2015a) und

Brisson et al. (2017) und wurden zur Beantwortung der dritten Fragestellung um den

Moderator und die Interaktionsterme ergänzt.

Konkret wurden pro abhängige Variable (d.h. Wertüberzeugungen und Leistung) und Moderator zum Posttest und zum Follow-Up separate Modelle spezifiziert. Alle Modelle enthielten auf Individual- sowie Klassenebene die korrespondierende Prätest-Variable des 
Outcomes sowie eine Moderatorvariable (d.h. Alltagsbezug, Lernunterstützung, enthusiastisches Verhalten) als Prädiktoren. Diese Variablen wurden auf der Individualebene am Klassenmittel zentriert (Enders und Tofighi, 2007) und auf Klassenebene manifest aggregiert (Marsh et al., 2009). Das jeweils interessierende Outcome wurde auf Klassenebene zudem auf zwei Dummy-Variablen, die die Zugehörigkeit zur jeweiligen Interventions- im Vergleich zur Kontrollgruppe anzeigten, regrediert. Darüber hinaus wurden zwei manifeste multiplikative Terme zwischen diesen Dummy-Variablen und dem entsprechenden Moderator auf Klassenebene als weitere Prädiktoren in das Modell aufgenommen. Mittels der Benjamini-Hochberg-Prozedur wurde eine Korrektur der $p$-Werte der Interaktionsterme vorgenommen (Benjamini und Hochberg, 1995).

2.4.3. Umgang mit fehlenden Werten. Fehlende Werte (zwischen 5,9\% und 12,0\%) wurden wie in vorherigen Überprüfungen der MoMa-Intervention (Gaspard et al., 2015a; Brisson et al., 2017) durch das Full Information Maximum Likelihood-Verfahren in Mplus berücksichtigt (Graham, 2009). Um die hierfür nötige Missing at Random-Annahme plausibler zu machen, wurden verschiedene zum Prätest erhobene Hilfsvariablen verwendet, indem Korrelationen dieser Variablen mit allen unabhängigen Variablen und mit den Fehlertermen der Outcome-Variablen sowie innerhalb der Hilfsvariablen spezifiziert wurden (s.g. Saturated Correlates Modell; siehe Collins et al., 2001; Graham, 2003). Diese Hilfsvariablen umfassten zu vorherigen Untersuchungen identische Variablen, d.h. das Geschlecht sowie mehrere Leistungsmaße (die durch die Schulen berichteten MathematikEndjahresnoten aus Klassenstufe 8 sowie - sofern nicht im Fokus der Analyse selbst Ergebnisse der Lernstandserhebung aus Klassenstufe 9 und das Ergebnis eines nonverbalen kognitiven Fähigkeitstests; Heller und Perleth, 2000). 


\section{Ergebnisse}

\subsection{Poweranalysen}

Zunächst interessierten uns die (1) MDESD bei einer Power von 0,80 und die (2)

MRSS zur Aufdeckung eines Moderationseffekts von 0,10 (bzw. 0,15) bei einer Power von 0,80. Hierfür wurden 54 Poweranalysen durchgeführt, deren Ergebnisse in Tab. 4 berichtet werden.

\section{Tabelle 4}

Minimale aufdeckbare Effektgrößendifferenz (MDESD) mit 95\%-Konfidenzintervall, sowie minimal erforderliche Stichprobengröße (MRSS) zur Aufdeckung von zu erwartenden Moderationseffekten $\left(\delta_{\text {Mod }}=0,10 \mathrm{bzw} . \delta_{\text {Mod }}=0,15\right)$ bei adäquater Power $(=0,80)$ je nach Interventionsbedingung

\begin{tabular}{|c|c|c|c|c|c|}
\hline & Nützlichkeit & Intrins. Wert & Wichtigkeit & Kosten & Leistung \\
\hline \multicolumn{6}{|c|}{ MDESD } \\
\hline \multicolumn{6}{|l|}{ Textbedingung } \\
\hline \multirow[t]{2}{*}{ Posttest } & 0,16 & 0,15 & 0,15 & 0,14 & - \\
\hline & {$[0,05 ; 0,27]$} & {$[0,04 ; 0,25]$} & {$[0,05 ; 0,26]$} & {$[0,04 ; 0,23]$} & - \\
\hline \multirow[t]{2}{*}{ Follow-Up } & 0,16 & 0,15 & 0,18 & 0,16 & 0,18 \\
\hline & {$[0,05 ; 0,27]$} & {$[0,04 ; 0,25]$} & {$[0,05 ; 0,31]$} & {$[0,05 ; 0,27]$} & {$[0,05 ; 0,30]$} \\
\hline \multicolumn{6}{|c|}{ Zitatebedingung } \\
\hline \multirow[t]{2}{*}{ Posttest } & 0,17 & 0,16 & 0,15 & 0,16 & - \\
\hline & {$[0,05 ; 0,29]$} & {$[0,05 ; 0,27]$} & {$[0,04 ; 0,25]$} & {$[0,05 ; 0,27]$} & - \\
\hline \multirow[t]{2}{*}{ Follow-Up } & 0,16 & 0,16 & 0,14 & 0,18 & 0,19 \\
\hline & {$[0,05 ; 0,28]$} & {$[0,05 ; 0,28]$} & {$[0,04 ; 0,24]$} & {$[0,05 ; 0,31]$} & {$[0,06 ; 0,32]$} \\
\hline \multicolumn{6}{|c|}{ MRSS } \\
\hline \multicolumn{6}{|l|}{$\begin{array}{l}\delta_{\text {Mod }}=0,10 \\
\quad \text { Textbedingung }\end{array}$} \\
\hline Posttest & 136 & 112 & 120 & 101 & - \\
\hline Follow-Up & 133 & 117 & 169 & 135 & 162 \\
\hline \multicolumn{6}{|c|}{ Zitatebedingung } \\
\hline Posttest & 141 & 118 & 105 & 118 & - \\
\hline Follow-Up & 127 & 125 & 97 & 154 & 169 \\
\hline \multicolumn{6}{|l|}{$\delta_{\mathrm{Mod}}=0,15$} \\
\hline Posttest & 64 & 54 & 57 & 49 & - \\
\hline Follow-Up & 63 & 56 & 79 & 64 & 76 \\
\hline \multicolumn{6}{|c|}{ Zitatebedingung } \\
\hline Posttest & 66 & 56 & 51 & 56 & - \\
\hline Follow-Up & 60 & 59 & 47 & 72 & 79 \\
\hline
\end{tabular}

Anmerkung. MDESD = minimale aufdeckbare Effektgrößendifferenz; MRSS = minimal erforderlicher Stichprobenumfang auf Klassenebene; $\delta_{\text {Mod }}=$ angenommener, zu erwartender Moderationseffekt. Die für die Durchführung der Poweranalysen relevanten Stichprobenparameter wurden post-hoc aus den Daten der MoMa-Studie ermittelt mit $\left.\mathrm{P}_{\text {Text }}=0,53 / \mathrm{P}_{\text {Zitate }}=0,48\right), \mathrm{g}^{*}=1$ und $n_{\text {Text }}=24 / n_{\text {Zitate }}=23$.

- = nicht zutreffend, da Leistung nur zum Follow-Up erhoben wurde. 
3.1.1. MDESD. Für eine exemplarische, auf Klassenebene randomisierte kontrollierte Feldstudie wie die MoMa-Studie fand sich für je eine Interventionsbedingung (im Vergleich zu Wartekontrollbedingung) zu beiden Testzeitpunkten eine MDESD zwischen 0,14 und 0,19 $(M d n=0,16)$. Darunter liegende (womöglich wahre) Moderationseffekte können nicht mit ausreichend hoher Wahrscheinlichkeit als signifikant angezeigt werden.

3.1.2. MRSS. Zur Aufdeckung eines Moderationseffekts von 0,10 müssten für je zwei Bedingungen zwischen 97 und 169 Klassen $(M d n=126)$ rekrutiert werden, um eine adäquate Power von 0,80 zu erreichen. Für die MoMa-Studie würde dies bei gleichmäßiger Verteilung auf die drei Bedingungen eine Gesamtstichprobe von etwa 189 Klassen bedeuten. Zur Aufdeckung eines Moderationseffekts von 0,15 müsste eine Gesamtstichprobe von etwa 89 Klassen rekrutiert werden.

Exemplarisch für Moderationseffekte der Textbedingung auf die Nützlichkeitswahrnehmung der Schülerinnen und Schüler zum Follow-Up kann in Abb. 1 das Verhältnis von Anzahl der Klassen zu Power betrachtet werden.

\section{Abbildung 1}

Power-Kurve für einen Moderationseffekt von $\delta_{\text {Mod }}=0,10$ der Textbedingung mit einem Klassenlevel-Moderator auf die Nützlichkeitswahrnehmung der Schülerinnen und Schüler zum Follow-Up in Abhängigkeit der Klassenanzahl J

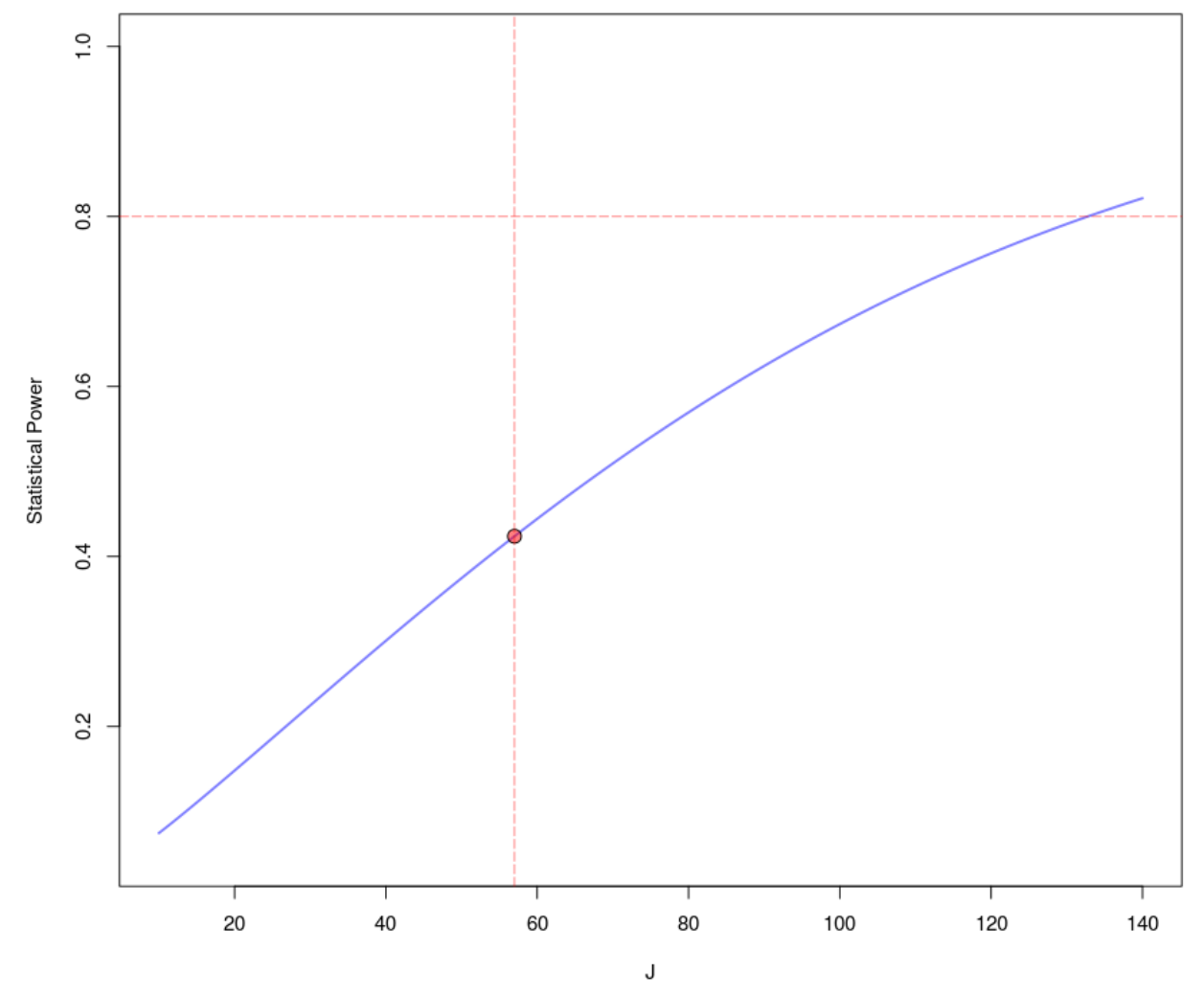




\subsection{Moderationsanalysen}

Die Ergebnisse der Mehrebenenregressionsanalysen finden sich in Tab. 5 bis 7.

Signifikante Ergebnisse sind darüber hinaus zur leichteren Interpretierbarkeit der Richtung in Abb. 2 visualisiert.

3.2.1. Haupteffekte der Moderatoren. Die Haupteffekte der Moderatoren geben den durchschnittlichen Effekt auf die entsprechenden Outcomes in der Wartekontrollbedingung an. Wie die Ergebnisse zeigen, hatte der wahrgenommene Alltagsbezug im Regelunterricht einen positiven Effekt auf die Wahrnehmung der Nützlichkeit und Wichtigkeit auf Individualebene zum Posttest sowie auf die Nützlichkeit auf Individual- und Klassenebene zum Follow-Up. Darüber hinaus erzielten Klassen, die einen höheren Alltagsbezug wahrnahmen, eine signifikant niedrigere Leistung unter zeitgleicher Kontrolle der Leistung zum Prätest. Auch für die wahrgenommene Lernunterstützung fand sich ein positiver Effekt auf die Nützlichkeit und die Wichtigkeit auf Individual- und Klassenebene (Wichtigkeit nur Posttest). Darüber hinaus verringerte eine hohe wahrgenommene Lernunterstützung die subjektiven Kosten auf Individualebene zum Posttest und zum Follow-Up und hatte eine negative Auswirkung auf die Leistung auf Klassenebene. Klassen, die das Verhalten ihrer Lehrkraft als enthusiastischer wahrnahmen, berichteten zudem eine höhere Nützlichkeitswahrnehmung und zeigten geringere Leistungen im Vergleich zum Prätest.

3.2.2. Moderationseffekte. Zusätzlich fanden sich in den beiden Interventionsbedingungen Interaktionseffekte zwischen $-0,38 \leq \beta \leq 0,12$ auf die Wertüberzeugungen der Schülerinnen und Schüler. Von diesen insgesamt 48 Moderationskoeffizienten wurden jedoch lediglich zehn (nach Korrektur der $p$-Werte sogar nur noch ein Koeffizient) als statistisch signifikant angezeigt: Es fanden sich keine signifikanten Interaktionen der Interventionsbedingungen mit dem wahrgenommenen Alltagsbezug. Im Gegensatz zum Alltagsbezug fanden sich in einigen Fällen jedoch signifikante Moderationseffekte mit der Lernunterstützung. Je höher die wahrgenommene 
Lernunterstützung der Lehrkraft durch die Klasse war, desto geringer war der Effekte beider Interventionsbedingungen auf die Nützlichkeitswahrnehmung (zum Posttest nur in der Zitatebedingung). Zugleich war die Intervention also auch umso wirksamer bzgl. der Nützlichkeitswahrnehmung, wenn die Lehrkraft als wenig lernunterstützend wahrgenommen wurde (insbesondere in der Zitatebedingung). Bezüglich der subjektiven Kosten verstärkte sich der Effekt beider Bedingungen zum Follow-Up, d.h. die Schülerinnen und Schüler berichteten weniger hohe Kosten, wenn die Lernunterstützung als höher wahrgenommen wurde. Ebenso wie bei der wahrgenommenen Lernunterstützung fanden sich auch bezüglich des wahrgenommenen enthusiastischen Verhaltens signifikante Moderationseffekte auf verschiedene Wertüberzeugungen der Schülerinnen und Schüler. Für Klassen, die das Verhalten der Lehrkraft als enthusiastischer wahrnahmen, war der Effekt der Zitatebedingung zum Follow-Up auf die Nützlichkeitswahrnehmung geringer. Zugleich fand sich sowohl zum Posttest als auch zum Follow-Up ein stärkerer negativer Effekt auf die Kosten (d.h. Schülerinnen und Schüler berichteten weniger hohe subjektive Kosten; zum Posttest nur in der Textbedingung). Zudem fand sich in der Textbedingung ein stärkerer positiver Effekt auf den intrinsischen Wert zum Posttest für Klassen, die das Verhalten der Lehrkraft als enthusiastischer wahrnahmen. 


\section{Tabelle 5}

Interventionseffekte in Abhängigkeit des durch die Schülerinnen und Schüler wahrgenommenen Alltagsbezugs zum Posttest und zum Follow-Up

\begin{tabular}{|c|c|c|c|c|c|c|c|c|c|c|c|c|c|c|c|c|c|c|c|c|}
\hline & \multicolumn{4}{|c|}{ Nützlichkeit } & \multicolumn{4}{|c|}{ Intrinsischer Wert } & \multicolumn{4}{|c|}{ Wichtigkeit } & \multicolumn{4}{|c|}{ Kosten } & \multicolumn{4}{|c|}{ Leistung } \\
\hline & Par. & $p$ & $p$ kor. & $(\mathrm{SE})$ & Par. & $p$ & $p$ kor. & $(\mathrm{SE})$ & Par. & $p$ & $p$ kor. & $(\mathrm{SE})$ & Par. & $p$ & $p$ kor. & (SE) & Par. & $p$ & $p$ kor. & (SE) \\
\hline \multicolumn{21}{|l|}{ Posttest } \\
\hline \multicolumn{21}{|l|}{ Individualebene ${ }^{\mathrm{a}}$} \\
\hline Wert zu T1 & 0,65 & 0,000 & & $(0,02)$ & 0,78 & 0,000 & & $(0,02)$ & 0,75 & 0,000 & & $(0,02)$ & 0,77 & 0,000 & & $(0,02)$ & & & & \\
\hline Alltagsbezug & 0,05 & 0,044 & & $(0,02)$ & 0,03 & 0,145 & & $(0,02)$ & 0,04 & 0,030 & & $(0,02)$ & $-0,02$ & 0,202 & & $(0,02)$ & & & & \\
\hline \multicolumn{21}{|l|}{ Klassenebene } \\
\hline Wert zu T1 & 0,73 & 0,000 & & $(0,07)$ & 0,77 & 0,000 & & $(0,07)$ & 0,72 & 0,000 & & $(0,08)$ & 0,82 & 0,000 & & $(0,07)$ & & & & \\
\hline Alltagsbezug & 0,12 & 0,062 & & $(0,07)$ & 0,07 & 0,323 & & $(0,07)$ & 0,01 & 0,815 & & $(0,06)$ & $-0,04$ & 0,512 & & $(0,06)$ & & & & \\
\hline Textbedingung ${ }^{\mathrm{b}}$ & 0,16 & 0,004 & & $(0,06)$ & 0,01 & 0,912 & & $(0,05)$ & 0,00 & 0,937 & & $(0,05)$ & $-0,02$ & 0,627 & & $(0,05)$ & & & & \\
\hline Zitatebedingung ${ }^{\mathrm{b}}$ & 0,30 & 0,000 & & $(0,06)$ & 0,09 & 0,108 & & $(0,05)$ & 0,12 & 0,029 & & $(0,05)$ & $-0,09$ & 0,113 & & $(0,06)$ & & & & \\
\hline Textb. $\times$ Alltagsbezug & $-0,04$ & 0,627 & 0,941 & $(0,09)$ & 0,09 & 0,271 & 0,665 & $(0,08)$ & 0,07 & 0,484 & 0,933 & $(0,09)$ & $-0,04$ & 0,534 & 0,901 & $(0,07)$ & & & & \\
\hline Zitateb. $\times$ Alltagsbezug & $-0,04$ & 0,678 & 0,915 & $(0,10)$ & $-0,08$ & 0,325 & 0,585 & $(0,08)$ & 0,09 & 0,421 & 0,632 & $(0,11)$ & 0,05 & 0,604 & 0,858 & $(0,09)$ & & & & \\
\hline \multicolumn{21}{|l|}{$\Delta R^{2}$} \\
\hline \multicolumn{21}{|l|}{ Individualebene } \\
\hline Alltagsbezug ${ }^{\mathrm{c}}$ & 0,00 & & & & 0,01 & & & & 0,00 & & & & 0,00 & & & & & & & \\
\hline \multicolumn{21}{|l|}{ Klassenebene } \\
\hline Alltagsbezug ${ }^{\mathrm{c}}$ & 0,01 & & & & 0,01 & & & & 0,01 & & & & 0,01 & & & & & & & \\
\hline Interaktionen $^{\mathrm{c}}$ & 0,00 & & & & 0,01 & & & & 0,00 & & & & 0,00 & & & & & & & \\
\hline \multicolumn{21}{|l|}{ Follow-Up } \\
\hline \multicolumn{21}{|l|}{ Individualebene ${ }^{\mathrm{a}}$} \\
\hline Wert zu T1 & 0,58 & 0,000 & & $(0,03)$ & 0,72 & 0,000 & & $(0,02)$ & 0,67 & 0,000 & & $(0,02)$ & 0,72 & 0,000 & & $(0,02)$ & 0,55 & 0,000 & & $(0,03)$ \\
\hline Alltagsbezug & 0,07 & 0,006 & & $(0,03)$ & 0,03 & 0,146 & & $(0,02)$ & 0,03 & 0,140 & & $(0,02)$ & 0,00 & 0,766 & & $(0,02)$ & 0,05 & 0,100 & & $(0,03)$ \\
\hline \multicolumn{21}{|l|}{ Klassenebene } \\
\hline Wert zu T1 & 0,73 & 0,000 & & $(0,08)$ & 0,76 & 0,000 & & $(0,08)$ & 0,75 & 0,000 & & $(0,09)$ & 0,87 & 0,000 & & $(0,09)$ & 0,33 & 0,000 & & $(0,07)$ \\
\hline Alltagsbezug & 0,14 & 0,024 & & $(0,06)$ & 0,04 & 0,586 & & $(0,08)$ & 0,04 & 0,561 & & $(0,07)$ & $-0,01$ & 0,933 & & $(0,09)$ & $-0,14$ & 0,048 & & $(0,07)$ \\
\hline Textbedingung ${ }^{\mathrm{b}}$ & 0,17 & 0,002 & & $(0,06)$ & 0,05 & 0,370 & & $(0,05)$ & 0,07 & 0,222 & & $(0,06)$ & $-0,01$ & 0,809 & & $(0,06)$ & 0,05 & 0,406 & & $(0,06)$ \\
\hline Zitatebedingung $^{\mathrm{b}}$ & 0,26 & 0,000 & & $(0,06)$ & 0,15 & 0,011 & & $(0,06)$ & 0,14 & 0,007 & & $(0,05)$ & $-0,06$ & 0,271 & & $(0,06)$ & 0,19 & 0,003 & & $(0,06)$ \\
\hline Textb. $\times$ Alltagsbezug & $-0,09$ & 0,323 & 0,727 & $(0,09)$ & 0,02 & 0,788 & 0,887 & $(0,09)$ & 0,04 & 0,758 & 0,975 & $(0,12)$ & $-0,06$ & 0,564 & 0,896 & $(0,10)$ & 0,06 & 0,514 & 0,925 & $(0,09)$ \\
\hline Zitateb. $\times$ Alltagsbezug & $-0,13$ & 0,209 & 0,470 & $(0,10)$ & $-0,09$ & 0,340 & 0,574 & $(0,10)$ & $-0,01$ & 0,944 & 0,980 & $(0,09)$ & 0,04 & 0,723 & 0,813 & $(0,12)$ & 0,04 & 0,714 & 0,876 & $(0,10)$ \\
\hline \multicolumn{21}{|l|}{$\Delta R^{2}$} \\
\hline \multicolumn{21}{|l|}{ Individualebene } \\
\hline Alltagsbezug ${ }^{\mathrm{c}}$ & 0,01 & & & & 0,00 & & & & 0,00 & & & & 0,00 & & & & 0,00 & & & \\
\hline \multicolumn{21}{|l|}{ Klassenebene } \\
\hline Alltagsbezug ${ }^{\mathrm{c}}$ & 0,01 & & & & 0,00 & & & & 0,01 & & & & 0,00 & & & & 0,06 & & & \\
\hline Interaktionen $^{\mathrm{c}}$ & 0,01 & & & & 0,01 & & & & 0,00 & & & & 0,00 & & & & 0,00 & & & \\
\hline
\end{tabular}


Anmerkung. $\quad \mathrm{T} 1=$ Prätest; Par. $=$ jeweiliger Parameter, d.h. entweder standardisierte Regressionskoeffizienten oder $\Delta R^{2} ; p=p$-Wert; $p$ kor. $=p$ Wert korrigiert nach Benjamini-Hochberg; $S E=$ Standardfehler. Als Hilfsvariablen wurden die Mathematiknote am Ende der Klassenstufe 8, das Ergebnis der Vergleichsarbeiten aus Klassenstufe 9 sowie das Geschlecht berücksichtigt. Interaktionen mit $p<0,05$ wurden jeweils fett gedruckt. ${ }^{a}$ Skalen wurden am Klassenmittel zentriert. Effekte auf Klassenebene lassen sich daher als Kompositionseffekte verstehen.

${ }^{\mathrm{b}}$ Effekte der Text- und der Zitatebedingung sind im Vergleich zur Wartekontrollbedingung zu verstehen. Beispielsweise bedeutet ein Effekt von 0,16 in der Textbedingung, dass der geschätzte Wert eines Schüler/einer Schülerin in der Textbedingung im Mittel um 0,16 SD höher ist als in der Wartekontrollbedingung zum gleichen Zeitpunkt.

${ }^{c}$ Die Ebenen-spezifischen Anteile zusätzlich erklärter Varianz durch die Hinzunahme der angegebenen Variablen (d.h. Alltagsbezug bzw. Interaktionen; $\Delta R_{w}^{2(f 1)}$ und $\Delta R_{b}^{2(f 2)}$ ) wurden nach Rights und Sterba (2020) bestimmt. Hierzu wurden neben den berichteten Modellen zwei weitere Modelle spezifiziert: Ein Modell, das nur den Prätestwert auf Individual- und Klassenebene sowie die zwei Interventionsbedingungen auf Klassenebene enthält; sowie ein weiteres Modell, das zusätzlich den Moderator (Alltagsbezug) auf Individual- und Klassenebene enthält. 


\section{Tabelle 6}

Interventionseffekte in Abhängigkeit der durch die Schülerinnen und Schüler wahrgenommenen Lernunterstützung durch die Lehrkraft zum

Posttest und zum Follow-Up

\begin{tabular}{|c|c|c|c|c|c|c|c|c|c|c|c|c|c|c|c|c|c|c|c|c|}
\hline & \multicolumn{4}{|c|}{ Nützlichkeit } & \multicolumn{4}{|c|}{ Intrinsischer Wert } & \multicolumn{4}{|c|}{ Wichtigkeit } & \multicolumn{4}{|c|}{ Kosten } & \multicolumn{4}{|c|}{ Leistung } \\
\hline & Par. & $p$ & $p$ kor. & $(\mathrm{SE})$ & Par. & $p$ & $p$ kor. & $(\mathrm{SE})$ & Par. & $p$ & $p$ kor. & $(\mathrm{SE})$ & Par. & $p$ & $p$ kor. & $(\mathrm{SE})$ & Par. & $p$ & $p$ kor. & $(\mathrm{SE})$ \\
\hline \multicolumn{21}{|l|}{ Posttest } \\
\hline \multicolumn{21}{|l|}{ Individualebene $\mathrm{a}^{\mathrm{a}}$} \\
\hline Wert zu T1 & 0,66 & 0,000 & & $(0,02)$ & 0,78 & 0,000 & & $(0,02)$ & 0,75 & 0,000 & & $(0,02)$ & 0,77 & 0,000 & & $(0,02)$ & & & & \\
\hline Lernunterstützung & 0,05 & 0,042 & & $(0,02)$ & $-0,01$ & 0,474 & & $(0,02)$ & 0,04 & 0,033 & & $(0,02)$ & $-0,04$ & 0,043 & & $(0,02)$ & & & & \\
\hline \multicolumn{21}{|l|}{ Klassenebene } \\
\hline Wert zu T1 & 0,75 & 0,000 & & $(0,06)$ & 0,76 & 0,000 & & $(0,07)$ & 0,70 & 0,000 & & $(0,08)$ & 0,78 & 0,000 & & $(0,07)$ & & & & \\
\hline Lernunterstützung & 0,25 & 0,007 & & $(0,09)$ & 0,06 & 0,411 & & $(0,08)$ & 0,14 & 0,021 & & $(0,06)$ & $-0,01$ & 0,820 & & $(0,06)$ & & & & \\
\hline Textbedingung ${ }^{\mathrm{b}}$ & 0,13 & 0,017 & & $(0,05)$ & $-0,01$ & 0,795 & & $(0,05)$ & $-0,02$ & 0,728 & & $(0,05)$ & $-0,02$ & 0,714 & & $(0,05)$ & & & & \\
\hline Zitatebedingung $^{\mathrm{b}}$ & 0,28 & 0,000 & & $(0,06)$ & 0,08 & 0,163 & & $(0,06)$ & 0,12 & 0,023 & & $(0,05)$ & $-0,08$ & 0,139 & & $(0,06)$ & & & & \\
\hline Textb. $\times$ Lernunterstützung & $-0,14$ & 0,198 & 0,668 & $(0,11)$ & 0,11 & 0,232 & 0,696 & $(0,09)$ & $-0,01$ & 0,877 & 0,877 & $(0,08)$ & $-0,08$ & 0,241 & 0,651 & $(0,07)$ & & & & \\
\hline Zitateb. $\times$ Lernunterstützung & $-0,28$ & 0,009 & 0,081 & $(0,11)$ & $-0,03$ & 0,715 & 0,839 & $(0,09)$ & $-0,15$ & 0,075 & 0,253 & $(0,08)$ & $-0,09$ & 0,225 & 0,467 & $(0,07)$ & & & & \\
\hline \multicolumn{21}{|l|}{$\Delta R^{2}$} \\
\hline \multicolumn{21}{|l|}{ Individualebene } \\
\hline Lernunterstützung $^{\mathrm{c}}$ & 0,00 & & & & 0,00 & & & & 0,00 & & & & 0,00 & & & & & & & \\
\hline \multicolumn{21}{|l|}{ Klassenebene } \\
\hline Lernunterstützung $^{\mathrm{c}}$ & 0,01 & & & & 0,01 & & & & 0,01 & & & & 0,03 & & & & & & & \\
\hline Interaktionen $^{\mathrm{c}}$ & 0,03 & & & & 0,01 & & & & 0,03 & & & & 0,01 & & & & & & & \\
\hline \multicolumn{21}{|l|}{ Follow-Up } \\
\hline \multicolumn{21}{|l|}{ Individualebene $\mathrm{a}^{\mathrm{a}}$} \\
\hline Wert zu T1 & 0,58 & 0,000 & & $(0,02)$ & 0,73 & 0,000 & & $(0,02)$ & 0,66 & 0,000 & & $(0,02)$ & 0,71 & 0,000 & & $(0,02)$ & 0,55 & 0,000 & & $(0,03)$ \\
\hline Lernunterstützung & 0,06 & 0,007 & & $(0,02)$ & 0,00 & 0,970 & & $(0,02)$ & 0,04 & 0,113 & & $(0,02)$ & $-0,06$ & 0,006 & & $(0,02)$ & 0,02 & 0,412 & & $(0,03)$ \\
\hline \multicolumn{21}{|l|}{ Klassenebene } \\
\hline Wert zu T1 & 0,77 & 0,000 & & $(0,07)$ & 0,73 & 0,000 & & $(0,08)$ & 0,73 & 0,000 & & $(0,08)$ & 0,82 & 0,000 & & $(0,09)$ & 0,31 & 0,000 & & $(0,07)$ \\
\hline Lernunterstützung & 0,28 & 0,000 & & $(0,07)$ & 0,06 & 0,288 & & $(0,06)$ & 0,16 & 0,101 & & $(0,10)$ & 0,12 & 0,149 & & $(0,09)$ & $-0,19$ & 0,011 & & $(0,07)$ \\
\hline Textbedingung ${ }^{\mathrm{b}}$ & 0,14 & 0,004 & & $(0,05)$ & 0,04 & 0,479 & & $(0,05)$ & 0,05 & 0,364 & & $(0,06)$ & $-0,03$ & 0,646 & & $(0,06)$ & 0,08 & 0,183 & & $(0,06)$ \\
\hline Zitatebedingung $^{\mathrm{b}}$ & 0,24 & 0,000 & & $(0,05)$ & 0,14 & 0,021 & & $(0,06)$ & 0,14 & 0,008 & & $(0,05)$ & $-0,08$ & 0,200 & & $(0,06)$ & 0,20 & 0,002 & & $(0,06)$ \\
\hline Textb. $\times$ Lernunterstützung & $-0,18$ & 0,044 & 0,238 & $(0,09)$ & 0,04 & 0,627 & 0,891 & $(0,08)$ & $-0,04$ & 0,773 & 0,907 & $(0,13)$ & $-0,22$ & $\mathbf{0 , 0 3 1}$ & 0,419 & $(0,10)$ & 0,17 & 0,096 & 0,370 & $(0,10)$ \\
\hline Zitateb. $\times$ Lernunterstützung & $-0,38$ & $\mathbf{0 , 0 0 0}$ & $\mathbf{0 , 0 0 0}$ & $(0,09)$ & $-0,03$ & 0,699 & 0,899 & $(0,08)$ & $-0,17$ & 0,122 & 0,299 & $(0,11)$ & $-0,22$ & $\mathbf{0 , 0 2 7}$ & 0,182 & $(0,10)$ & 0,15 & 0,077 & 0,231 & $(0,09)$ \\
\hline \multicolumn{21}{|l|}{$\Delta R^{2}$} \\
\hline \multicolumn{21}{|l|}{ Individualebene } \\
\hline Lernunterstützung $^{\mathrm{c}}$ & 0,00 & & & & 0,00 & & & & 0,00 & & & & 0,00 & & & & 0,00 & & & \\
\hline \multicolumn{21}{|l|}{ Klassenebene } \\
\hline Lernunterstützung $^{\mathrm{c}}$ & 0,00 & & & & 0,01 & & & & 0,01 & & & & 0,00 & & & & 0,02 & & & \\
\hline Interaktionen $^{\mathrm{c}}$ & 0,06 & & & & 0,00 & & & & 0,01 & & & & 0,03 & & & & 0,02 & & & \\
\hline
\end{tabular}


Anmerkung. $\quad \mathrm{T} 1=$ Prätest; Par. $=$ jeweiliger Parameter, d.h. entweder standardisierte Regressionskoeffizienten oder $\Delta R^{2} ; p=p$-Wert; $p$ kor. $=p$ Wert korrigiert nach Benjamini-Hochberg; $S E=$ Standardfehler. Als Hilfsvariablen wurden die Mathematiknote am Ende der Klassenstufe 8, das Ergebnis der Vergleichsarbeiten aus Klassenstufe 9 sowie das Geschlecht berücksichtigt. Interaktionen mit $p<0,05$ wurden jeweils fett gedruckt. ${ }^{a}$ Skalen wurden am Klassenmittel zentriert. Effekte auf Klassenebene lassen sich daher als Kompositionseffekte verstehen.

${ }^{\mathrm{b}}$ Effekte der Text- und der Zitatebedingung sind im Vergleich zur Wartekontrollbedingung zu verstehen. Beispielsweise bedeutet ein Effekt von 0,13 in der Textbedingung, dass der geschätzte Wert eines Schüler/einer Schülerin in der Textbedingung im Mittel um 0,13 SD höher ist als in der Wartekontrollbedingung zum gleichen Zeitpunkt.

${ }^{\mathrm{c}}$ Die Ebenen-spezifischen Anteile zusätzlich erklärter Varianz durch die Hinzunahme der angegebenen Variablen (d.h. Lernunterstïtzung bzw. Interaktionen; $\Delta R_{w}^{2(f 1)}$ und $\Delta R_{b}^{2(f 2)}$ ) wurden nach Rights und Sterba (2020) bestimmt. Hierzu wurden neben den berichteten Modellen zwei weitere Modelle spezifiziert: Ein Modell, das nur den Prätestwert auf Individual- und Klassenebene sowie die zwei Interventionsbedingungen auf Klassenebene enthält; sowie ein weiteres Modell, das zusätzlich den Moderator (Lernunterstützung) auf Individual- und Klassenebene enthält. 


\section{Tabelle 7}

Interventionseffekte in Abhängigkeit des durch die Schülerinnen und Schüler wahrgenommenen enthusiastischen Verhaltens der Lehrkraft zum

Posttest und zum Follow-Up

\begin{tabular}{|c|c|c|c|c|c|c|c|c|c|c|c|c|c|c|c|c|c|c|c|c|}
\hline & \multicolumn{4}{|c|}{ Nützlichkeit } & \multicolumn{4}{|c|}{ Intrinsischer Wert } & \multicolumn{4}{|c|}{ Wichtigkeit } & \multicolumn{4}{|c|}{ Kosten } & \multicolumn{4}{|c|}{ Leistung } \\
\hline & Par. & $p$ & $p$ kor. & $(\mathrm{SE})$ & Par. & $p$ & $p$ kor. & $(\mathrm{SE})$ & Par. & $p$ & $p$ kor. & $(\mathrm{SE})$ & Par. & $p$ & $p$ kor. & $(\mathrm{SE})$ & Par. & $p$ & $p$ kor. & $(\mathrm{SE})$ \\
\hline \multicolumn{21}{|l|}{ Posttest } \\
\hline \multicolumn{21}{|l|}{ Individualebene $^{\mathrm{a}}$} \\
\hline Wert zu T1 & 0,66 & 0,000 & & $(0,02)$ & 0,78 & 0,000 & & $(0,02)$ & 0,75 & 0,000 & & $(0,02)$ & 0,77 & 0,000 & & $(0,02)$ & & & & \\
\hline Enthusiasmus & 0,01 & 0,615 & & $(0,02)$ & 0,02 & 0,413 & & $(0,02)$ & 0,02 & 0,373 & & $(0,02)$ & $-0,02$ & 0,179 & & $(0,02)$ & & & & \\
\hline \multicolumn{21}{|l|}{ Klassenebene } \\
\hline Wert zu T1 & 0,72 & 0,000 & & $(0,07)$ & 0,73 & 0,000 & & $(0,07)$ & 0,67 & 0,000 & & $(0,08)$ & 0,77 & 0,000 & & $(0,07)$ & & & & \\
\hline Enthusiastisches Verhalten & 0,16 & 0,047 & & $(0,08)$ & 0,06 & 0,305 & & $(0,06)$ & 0,09 & 0,088 & & $(0,06)$ & 0,03 & 0,573 & & $(0,06)$ & & & & \\
\hline Textbedingung ${ }^{\mathrm{b}}$ & 0,14 & 0,010 & & $(0,05)$ & $-0,01$ & 0,864 & & $(0,05)$ & $-0,01$ & 0,823 & & $(0,05)$ & $-0,02$ & 0,599 & & $(0,04)$ & & & & \\
\hline Zitatebedingung & 0,29 & 0,000 & & $(0,06)$ & 0,08 & 0,122 & & $(0,05)$ & 0,12 & 0,016 & & $(0,05)$ & $-0,09$ & 0,115 & & $(0,06)$ & & & & \\
\hline Textb. $\times$ Enth. Verhalten & $-0,03$ & 0,768 & 0,943 & $(0,09)$ & $\mathbf{0 , 1 2}$ & 0,049 & 0,221 & $(0,06)$ & 0,07 & 0,368 & 0,764 & $(0,07)$ & $-0,13$ & $\mathbf{0 , 0 3 2}$ & 0,288 & $(0,06)$ & & & & \\
\hline Zitateb. $\times$ Enth. Verhalten & $-0,16$ & 0,089 & 0,240 & $(0,09)$ & $-0,01$ & 0,822 & 0,888 & $(0,07)$ & $-0,08$ & 0,242 & 0,467 & $(0,07)$ & $-0,13$ & 0,065 & 0,251 & $(0,07)$ & & & & \\
\hline \multicolumn{21}{|l|}{$\Delta R^{2}$} \\
\hline \multicolumn{21}{|l|}{ Individualebene } \\
\hline Enthusiastisches Verhalten ${ }^{c}$ & 0,00 & & & & 0,00 & & & & 0,00 & & & & 0,00 & & & & & & & \\
\hline \multicolumn{21}{|l|}{ Klassenebene } \\
\hline Enthusiastisches Verhalten ${ }^{c}$ & 0,02 & & & & 0,02 & & & & 0,04 & & & & 0,03 & & & & & & & \\
\hline Interaktionen $^{\mathrm{c}}$ & 0,02 & & & & 0,02 & & & & 0,01 & & & & 0,02 & & & & & & & \\
\hline \multicolumn{21}{|l|}{ Follow-Up } \\
\hline \multicolumn{21}{|l|}{ Individualebene ${ }^{\mathrm{a}}$} \\
\hline Wert zu T1 & 0,60 & 0,000 & & $(0,02)$ & 0,73 & 0,000 & & $(0,02)$ & 0,67 & 0,000 & & $(0,02)$ & 0,72 & 0,000 & & $(0,02)$ & 0,55 & 0,000 & & $(0,03)$ \\
\hline Enthusiasmus & $-0,02$ & 0,467 & & $(0,02)$ & 0,01 & 0,633 & & $(0,03)$ & 0,00 & 0,931 & & $(0,02)$ & $-0,03$ & 0,129 & & $(0,02)$ & 0,02 & 0,634 & & $(0,03)$ \\
\hline \multicolumn{21}{|l|}{ Klassenebene } \\
\hline Wert zu T1 & 0,73 & 0,000 & & $(0,08)$ & 0,71 & 0,000 & & $(0,08)$ & 0,69 & 0,000 & & $(0,09)$ & 0,84 & 0,000 & & $(0,09)$ & 0,34 & 0,000 & & $(0,06)$ \\
\hline Enthusiasmus & 0,18 & 0,014 & & $(0,07)$ & 0,07 & 0,182 & & $(0,06)$ & 0,12 & 0,154 & & $(0,08)$ & 0,09 & 0,166 & & $(0,06)$ & $-0,24$ & 0,000 & & $(0,06)$ \\
\hline Textbedingung ${ }^{\mathrm{b}}$ & 0,15 & 0,003 & & $(0,05)$ & 0,04 & 0,436 & & $(0,05)$ & 0,06 & 0,309 & & $(0,06)$ & $-0,02$ & 0,700 & & $(0,06)$ & 0,08 & 0,146 & & $(0,06)$ \\
\hline Zitatebedingung ${ }^{\mathrm{b}}$ & 0,26 & 0,000 & & $(0,05)$ & 0,13 & 0,017 & & $(0,06)$ & 0,14 & 0,007 & & $(0,05)$ & $-0,07$ & 0,235 & & $(0,06)$ & 0,21 & 0,000 & & $(0,06)$ \\
\hline Textb. $\times$ Enthusiasmus & $-0,04$ & 0,698 & 0,942 & $(0,09)$ & 0,01 & 0,850 & 0,918 & $(0,07)$ & 0,02 & 0,862 & 0,895 & $(0,10)$ & $-0,15$ & $\mathbf{0 , 0 3 7}$ & 0,250 & $(0,07)$ & 0,19 & $\mathbf{0 , 0 2 7}$ & 0,729 & $(0,09)$ \\
\hline Zitateb. $\times$ Enthusiasmus & $-0,27$ & $\mathbf{0 , 0 0 5}$ & 0,068 & $(0,10)$ & 0,00 & 0,972 & 0,972 & $(0,08)$ & $-0,09$ & 0,384 & 0,610 & $(0,10)$ & $-0,15$ & 0,049 & 0,265 & $(0,08)$ & 0,16 & 0,060 & 0,270 & $(0,09)$ \\
\hline \multicolumn{21}{|l|}{$\Delta R^{2}$} \\
\hline \multicolumn{21}{|l|}{ Individualebene } \\
\hline Enthusiastisches Verhalten $^{c}$ & 0,00 & & & & 0,00 & & & & 0,00 & & & & 0,00 & & & & 0,00 & & & \\
\hline \multicolumn{21}{|l|}{ Klassenebene } \\
\hline Enthusiastisches Verhalten $^{\mathrm{c}}$ & 0,02 & & & & 0,02 & & & & 0,03 & & & & 0,00 & & & & 0,08 & & & \\
\hline Interaktionen $^{\mathrm{c}}$ & 0,05 & & & & 0,00 & & & & 0,01 & & & & 0,01 & & & & 0,05 & & & \\
\hline
\end{tabular}


Anmerkung. $\quad \mathrm{T} 1=$ Prätest; Par. $=$ jeweiliger Parameter, d.h. entweder standardisierte Regressionskoeffizienten oder $\Delta R^{2} ; p=p$-Wert; $p$ kor. $=p$ Wert korrigiert nach Benjamini-Hochberg; $S E=$ Standardfehler. Als Hilfsvariablen wurden die Mathematiknote am Ende der Klassenstufe 8, das Ergebnis der Vergleichsarbeiten aus Klassenstufe 9 sowie das Geschlecht berücksichtigt. Interaktionen mit $p<0,05$ wurden jeweils fett gedruckt. ${ }^{a}$ Skalen wurden am Klassenmittel zentriert. Effekte auf Klassenebene lassen sich daher als Kompositionseffekte verstehen.

${ }^{\mathrm{b}}$ Effekte der Text- und der Zitatebedingung sind im Vergleich zur Wartekontrollbedingung zu verstehen. Beispielsweise bedeutet ein Effekt von 0,14 in der Textbedingung, dass der geschätzte Wert eines Schüler/einer Schülerin in der Textbedingung im Mittel um 0,14 SD höher ist als in der Wartekontrollbedingung zum gleichen Zeitpunkt.

${ }^{c}$ Die Ebenen-spezifischen Anteile zusätzlich erklärter Varianz durch die Hinzunahme der angegebenen Variablen (d.h. enthusiastisches Verhalten bzw. Interaktionen; $\Delta R_{w}^{2(f 1)}$ und $\Delta R_{b}^{2(f 2)}$ ) wurden nach Rights und Sterba (2020) bestimmt. Hierzu wurden neben den berichteten Modellen zwei weitere Modelle spezifiziert: Ein Modell, das nur den Prätestwert auf Individual- und Klassenebene sowie die zwei Interventionsbedingungen auf Klassenebene enthält; sowie ein weiteres Modell, das zusätzlich den Moderator (enthusiastisches Verhalten) auf Individual- und Klassenebene enthält. 


\section{Abbildung 2}

Darstellung adjustierter Mittelwerte aus den 8 (von 27 getesteten) Modellen, in denen Interaktionen der Interventionsbedingungen mit den motivationalen Unterrichtspraktiken als signifikant angezeigt wurden. Die adjustierten Mittelwerte sind getrennt für Klassen mit einer unterdurchschnittlichen (-1 SD) bzw. ̈̈berdurchschnittlichen (+1 SD) Ausprägung motivationaler Unterrichtspraktiken dargestellt.
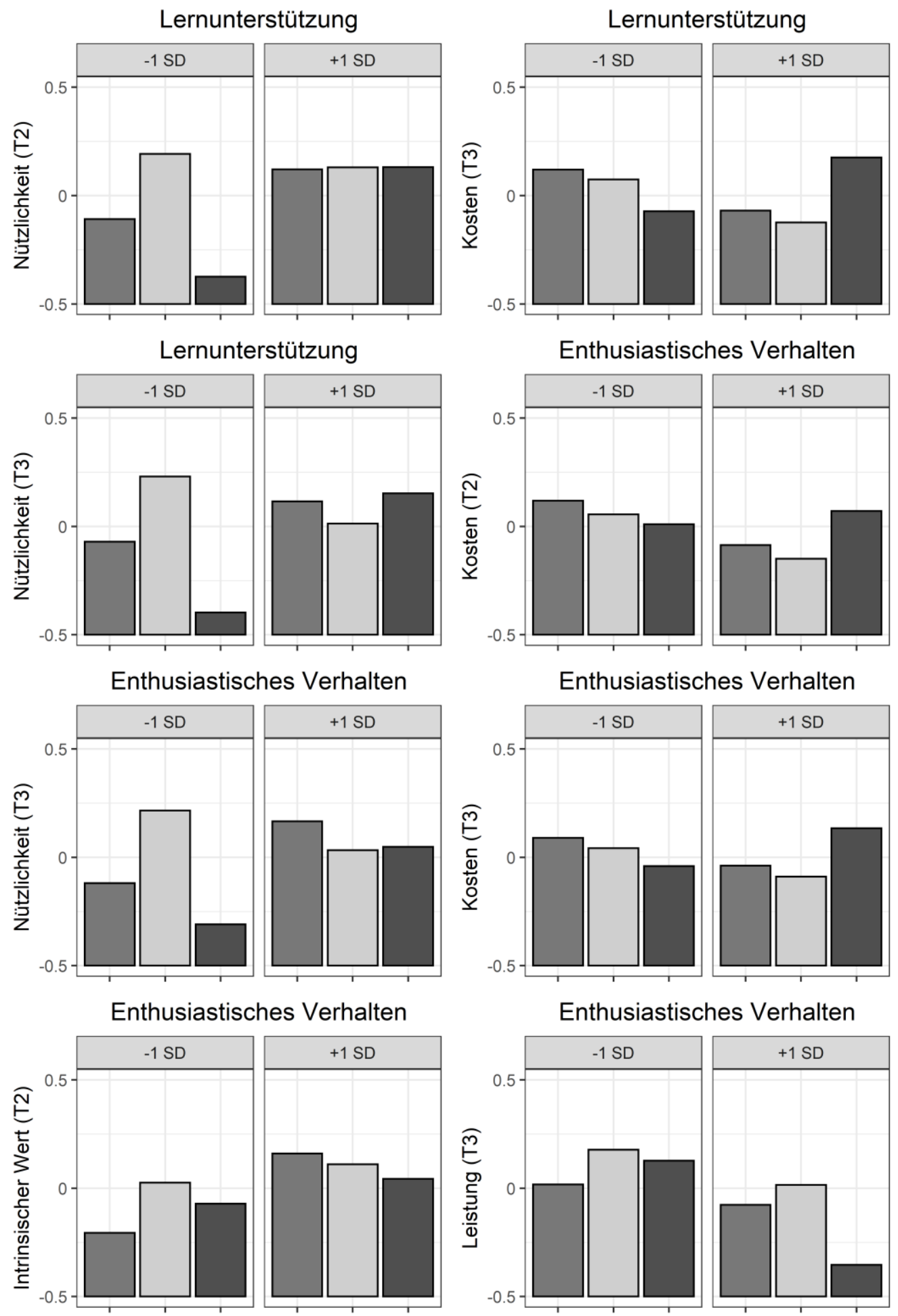

Bedingung $\square$ Text $\square$ Zitate $\square$ WKG 
Bezüglich der Leistung fanden sich ausschließlich positive Moderationseffekte der beiden Interventionsbedingungen mit den drei Moderatoren $(0,04 \leq \beta \leq 0,19)$. In einem von sechs Fällen (nach Korrektur der $p$-Werte sogar in keinem) wurden diese statistisch signifikant. Für Schülerinnen und Schüler, die das Verhalten der Lehrkraft im Vergleich zum Klassenmittel als enthusiastischer wahrnahmen, fand sich ein größerer Effekt der Textbedingung auf ihre Leistungen.

\section{Diskussion}

In den letzten Jahren wurden vermehrt Interventionen entwickelt, um die Motivation von Schülerinnen und Schülern zu fördern, und auf ihre Wirksamkeit getestet (für einen Überblick siehe Lazowski und Hulleman, 2016). Dabei ist eine offene Frage, ob die Wirksamkeit einer Intervention auch durch den motivationalen Unterrichtskontext, in den die Intervention eingebettet ist, beeinflusst sein kann. In Einklang mit vorheriger Forschung fanden wir Zusammenhänge motivationaler Unterrichtspraktiken (Alltagsbezug im Mathematikunterricht, Lernunterstützung durch die Lehrkraft sowie enthusiastisches Verhalten der Lehrkraft) mit den Wertüberzeugungen der Schülerinnen und Schüler (z.B. Frenzel et al., 2010; Kunter et al., 2013; Schmidt et al., 2019). Der vorliegenden Untersuchung lag jedoch die Frage zugrunde, ob der Erfolg einer gezielten, von außen an eine Klasse herangetragenen Motivationsintervention von solchen vielversprechenden Unterrichtspraktiken zur Förderung der Schülerinnen- und Schülermotivation abhängt.

Problematisch bei der Untersuchung solcher Moderationseffekte in Abhängigkeit des Kontextes sind Anforderungen an die Stichprobengröße. Wie unsere Ergebnisse zeigten, ist die Power der MoMa-Studie - einem großen CRT mit Schülerinnen und Schülern der neunten Jahrgangsstufe - lediglich ausreichend, Moderationseffekte auf die Wertüberzeugungen und die Leistung der Schülerinnen und Schüler ab einer Effektgröße von etwa 0,16 mit einer Teststärke von 0,80 aufzudecken. Die Wahrscheinlichkeit, einen tatsächlich vorhandenen, aber kleineren Moderationseffekt als statistisch signifikant anzuzeigen, ist folglich gering. Für 
mit der MoMa-Studie vergleichbare randomisierte kontrollierte Feldstudien impliziert dies, dass unter üblichen bis idealen Bedingungen (d.h. Planung der Studie bereits auf Basis einer a priori Poweranalyse zur Aufdeckung von relativ geringen Haupteffekten) lediglich verhältnismäßig große wahre Moderationseffekte mit einer akzeptablen Power aufgedeckt werden können. Oftmals fallen Moderationseffekte in der Praxis jedoch vermutlich kleiner aus, was ihre Aufdeckung unter vergleichbaren Bedingungen deutlich erschwert. Für die Planung einer auf Klassenebene randomisierten kontrollierten Feldstudie mit vergleichbaren Parametern wie die der MoMa-Studie wären zur Aufdeckung eines vermutlich realistischeren Moderationseffekts von 0,10 (bzw. 0,15, was aber wohl eher als obere Grenze für erwartbare Moderationseffekte anzunehmen ist) mindestens 189 (bzw. 89 Klassen) statt tatsächlichen 82 Klassen notwendig.

Bei der Untersuchung von Interventionseffekten der MoMa-Intervention auf die Nützlichkeit, intrinsischen Wert, Wichtigkeit, Kosten und Leistungen in Abhängigkeit des durch die Schülerinnen und Schüler wahrgenommenen Alltagsbezugs, Lernunterstützung und enthusiastischen Verhaltens der Lehrkraft fanden sich folgerichtig nur wenige, eher unsystematische Moderationseffekte, die mit Vorsicht interpretiert werden müssen. Insgesamt wurden elf der insgesamt 54 getesteten Interaktionsterme (20,37 \%) und nach Korrektur der p-Werte nach Benjamini und Hochberg (1995) sogar nur noch ein Term $(1,85 \%)$ als statistisch signifikant angezeigt. Darunter fanden sich in Abhängigkeit der wahrgenommenen Lernunterstützung und des enthusiastischen Verhaltens geringere Interventionseffekte auf die Nützlichkeitswahrnehmung und zum Teil stärkere Effekte auf die subjektiven Kosten und Leistung. Neben den gefunden signifikanten Moderationseffekten könnten weitere wahre Effekte vorliegen, diese aufgrund ihrer Größe jedoch nicht als statistisch signifikant angezeigt werden. Zugleich ist die Höhe der als signifikant angezeigten Moderationseffekte nicht unbedingt interpretierbar, da diese bei zu geringer Power oftmals tatsächliche Effekte 
überschätzen (Gelman und Carlin, 2014). Die folgenden Schlussfolgerungen sind daher unter Berücksichtigung der beschriebenen Einschränkungen zu interpretieren und einzuordnen.

Es scheint, als mache es bezüglich der Nützlichkeitswahrnehmung von Schülerinnen und Schülern, deren Lehrkräfte motivationale Unterrichtspraktiken wie die Lernunterstützung oder ein enthusiastisches Auftreten im Unterricht verwenden, keine großen Unterschiede, ob sie die MoMa-Intervention erhielten oder nicht. Ihre Nützlichkeitswahrnehmung entwickelte sich im Laufe des Schuljahres ähnlich zu den Mitschülerinnen und -schülern, die keine Intervention erhielten (siehe auch linke Hälfte von Abb. 2). Je geringer diese motivationalen Ressourcen jedoch waren, desto relevanter erschien der Erhalt der MoMa-Intervention, da ihre Auswirkung auf die Nützlichkeitswahrnehmung stieg. In der Tendenz schien dies auch für die Wichtigkeit und den intrinsischen Wert zuzutreffen, ohne dass sich hierfür signifikante Hinweise finden.

Diese Befunde deuten auf einen Einfluss des motivationalen Kontexts hin, der sich eher aus einer defizitären Perspektive erklären ließe (Rosenzweig und Wigfield, 2016). Lehrkräfte können ihre Schülerinnen und Schüler durch Lernunterstützung und mit enthusiastischem Verhalten für ein Fach motivieren (z.B. Dietrich et al., 2015; Frenzel et al., 2018). Werden Schülerinnen und Schüler hingegen im Regelunterricht nicht hinreichend dabei unterstützt, Freude an und Motivation für Mathematik zu entdecken und aufrechtzuerhalten, kann eine zielgerichtete Intervention ein Schlüsselmoment sein, das die Motivation der Schülerinnen und Schüler umso stärker beeinflusst.

Bezüglich der subjektiven Kosten und der Leistung der Schülerinnen und Schüler fanden wir entgegengesetzte Hinweise zur Wirkweise der motivationalen Unterrichtspraktiken, die somit eher auf eine Potenzialperspektive hindeuteten (siehe auch rechte Hälfte von Abb. 2; siehe außerdem Walton und Yeager, 2020). Je positiver der wahrgenommene motivationale Unterrichtskontext (d.h. Lernunterstützung und enthusiastisches Verhalten) durch die Lehrkraft im Regelunterricht, desto stärker führte die 
MoMa-Intervention scheinbar zu niedrigeren Kosten und besseren Leistungen (insbesondere in der Textbedingung).

Entgegen unserer Erwartungen fanden sich keine differenziellen Effekte der MoMaIntervention in Abhängigkeit des Alltagsbezugs im Regelunterricht. Dies könnte zum einen auf die fehlende Power zurückführbar sein. Zum anderen könnte es sein, dass eine so stark kognitiv beurteilte Kontextvariable wie der Alltagsbezug (entgegen der Lernunterstützung und dem enthusiastischen Verhalten; Frenzel et al., 2010), der darüber hinaus mit der individuellen Nützlichkeitswahrnehmung konfundiert sein könnte, tatsächlich zu keiner unterschiedlichen Wirksamkeit der MoMa-Intervention führt.

\subsection{Stärken, Grenzen und zukünftige Forschung}

Die Untersuchung von kontextuellen differenziellen Effekten einer Motivationsintervention ist bislang keine gängige Praxis, auch wenn sie aufschlussreiche Hinweise über die Heterogenität von Interventionseffekten und die mögliche flächendeckende Wirksamkeit von Interventionen liefern würden. Bislang geraten zwar vereinzelt kontextuelle Merkmale in den Fokus von Untersuchungen wie bspw. die Einstellungen der Mitschülerinnen und Mitschüler (Yeager et al., 2019), die aktuelle Studie ist jedoch die erste uns bekannte, die motivationale Unterrichtspraktiken der Lehrkraft im Regelunterricht als mögliche Moderatoren der Wirksamkeit einer Motivationsintervention im Klassenkontext untersucht. Dabei wurden potenzielle Moderatoren betrachtet, die aus der bisherigen Forschung abgeleitet als vielversprechende Einflussfaktoren auf die Motivation von Schülerinnen und Schüler zu bewerten sind.

Unklar ist dabei jedoch, welche Effektgrößen bei der Betrachtung von Moderationseffekten zu erwarten sind. Die Stichprobenparameter, die für die Durchführung einer Poweranalyse notwendig sind, wurden für diese Untersuchung direkt aus der MoMaStudie ermittelt. Im optimalen Fall einer a priori durchgeführten Poweranalyse müssen entsprechende Parameter (bspw. ICC der interessierenden Outcome-Variable) jedoch aus 
vorheriger Forschung abgeleitet werden - hierfür sind realistische Richtlinien (empirical benchmarks; Hill et al., 2008), die auf Analysen mit möglichst ähnlichen Studien beruhen, notwendig. Bezüglich der Bewertung realistischer Effektgrößen ist zudem zu berücksichtigen, ob diese auch als substantiell einzuordnen sind - hierfür ist die Orientierung an der Höhe möglicher Effekte auf die Motivation von Schülerinnen und Schülern innerhalb eines Schuljahres als „maximal erreichbare Effektgröße“ denkbar (Yeager et al., 2019; siehe auch Hanushek, 2011; Funder und Ozer, 2019). Erste Anhaltspunkte hierfür liefern zwar beispielsweise Brunner und Kollegen (2018), die Design-Parameter von repräsentativen PISA-Daten bezüglich Leistung, Affekt und Motivation sowie Lernstrategien von 15-jährigen Schülerinnen und Schülern aus 81 Ländern präsentieren, aber weitere richtungsweisende Untersuchungen insbesondere im deutschsprachigen Raum sind wünschenswert.

Wie die Poweranalysen der vorliegenden Untersuchung zudem zeigten, ist die MoMaStudie mit zu wenigen Klassen ausgestattet, um kleine Moderationseffekte mit ausreichend großer Wahrscheinlichkeit aufdecken zu können. Es ist also unklar, ob die gefundenen Moderationseffekte bereits alle wahren Effekte abdecken und ob signifikante Moderationseffekte die wahren Effekte überschätzen. Eine Replikation der MoMa-Studie mit adäquater Stichprobengröße auf Klassenebene zur Aufdeckung kleiner Moderationseffekte wäre folglich notwendig (aber siehe bspw. Stallasch et al., 2021).

\subsection{Forschungspraktische Implikationen}

Auch wenn die Suche nach bedeutsamen Moderatoren von großem inhaltlichen Interesse ist, stößt sie jedoch forschungspraktisch und ökonomisch oftmals an ihre Grenzen (Moerbeek, 2006). Unsere Ergebnisse zeigen deutlich, dass das Testen von

Moderationseffekten im Rahmen einer Interventionsstudie, die nicht für diesen Zweck geplant wurde, mit großen Schwierigkeiten verbunden sein kann und signifikante Moderationseffekte daher mit Vorsicht interpretiert werden müssen. Stattdessen könnte also die entscheidende Schlussfolgerung für zukünftige Interventionsstudien die Notwendigkeit sein, direkt bei der 
Planung mögliche Moderationsfragestellungen zu berücksichtigen und die Rekrutierung entsprechend auszulegen. Für die Interventionsforschung wird aktuell stark diskutiert, unter welchen Bedingungen Interventionen im Klassenkontext erfolgreich sind (bspw. Yeager et al., 2019). Umso mehr besteht also der Bedarf nach genügend groß angelegten Studien, die Antworten auf die Interaktion mit individuellen und ebenso kontextuellen Faktoren liefern können. Diese Untersuchung konnte erste Hinweise darauf liefern, dass eine Nützlichkeitsintervention wie die MoMa-Intervention in motivational-defizitären Unterrichtskontexten, in denen also typischerweise ein höherer Förderbedarf besteht, besonders wirksam die Nützlichkeitswahrnehmung von Schülerinnen und Schülern fördern kann. Weiterführende Forschung in diese Richtung erachten wir als besonders vielversprechend.

Die Rekrutierung von adäquaten Stichproben, die eine auf Klassenebene randomisierte kontrollierte Feldstudie mit ausreichender Power zur Aufdeckung von Moderationseffekten ausstatten, geht jedoch mit immensem finanziellen und zeitlichen Aufwand einher. Es erscheint folglich eher unrealistisch, in Zukunft immer größere Stichproben zu rekrutieren, deren Größe auch die Aufdeckung von geringfügigen Moderationseffekten zulässt. Fraglich ist somit, ob die Untersuchung solcher Moderationseffekte die hohen Kosten, die damit einhergingen, rechtfertigt. Joyce und Cartwright (2020) fordern daher anstelle der Durchführung großangelegter Evaluationsstudien, deren Ergebnisse Rückschlüsse auf eine Population ermöglichen, die Evaluation von Interventionen jeweils unter gegebenen Umständen in gegebenen Subpopulationen - ohne den Anspruch der Verallgemeinerbarkeit. Entsprechend wäre es nicht erstrebenswert, immer größere Studien zu planen, die die Untersuchung von Moderatoren erlauben, sondern vielmehr gezielte Untersuchungen von Interventionen in bestimmten Kontexten, wobei die Interventionen den Spezifika des Kontexts vorab angepasst werden sollten. 


\subsection{Praktische Implikationen}

Praktische Implikationen lassen sich aus dieser Untersuchung aufgrund der geringen Power zur Aufdeckung von differenziellen Effekten nur mit Vorsicht ziehen. Nichtsdestotrotz lässt sich aufgrund der Ergebnisse zumindest schlussfolgern, dass Merkmale auf Klassenebene durchaus Einfluss auf die Wirksamkeit einer Motivationsintervention haben können. Nicht in jedem Klassenzimmer ist eine solche Intervention immer gleichermaßen wirksam. Eine Motivationsintervention, die sich in einem gewissen Kontext, in einer bestimmten Klasse oder bei einer bestimmten Lehrkraft als erfolgreich herausgestellt hat, kann keine Wirkung oder - im Extremfall - sogar eine negative Wirkung in einem anderen Kontext, einer anderen Klasse oder bei einer anderen Lehrkraft aufweisen. Dies sollte vor dem Einsatz einer sich im Mittel als wirksam erwiesenen Intervention berücksichtigt werden. Die MoMa-Intervention zeigte sich im unterdurchschnittlichen, durchschnittlichen, oder überdurchschnittlichen motivationalen Unterrichtskontext als weitestgehend förderlich oder im schlimmsten Fall als nicht schädlich. Somit kann der Einsatz der MoMa-Intervention unter Berücksichtigung der untersuchten Konstrukte insgesamt als positiv bewertet werden.

\subsection{Fazit}

Die Frage „Für wen und unter welchen Umständen ist eine Intervention wirksam?““(Spybrook et al., 2016, S. 606) bleibt oftmals unbeantwortet. Ein Grund sind methodische Herausforderungen bei der Aufdeckung solcher Moderationseffekte. Anhand einer exemplarischen, auf Klassenebene randomisierten kontrollierten Feldstudie, der MoMaStudie, konnten wir zeigen, dass zur Aufdeckung von erwartungsgemäß geringen Moderationseffekten eine vielfach größere Anzahl an Klassen notwendig ist als zur Aufdeckung von Haupteffekten einer Intervention. Diese Studie liefert außerdem erste Hinweise darauf, dass Motivationsinterventionen im Klassenkontext in Abhängigkeit von motivationalen Unterrichtspraktiken unterschiedlich wirksam sein können. Weitere Studien mit ausreichender Power wären jedoch notwendig, um diese ersten Hinweise zu bekräftigen. 


\section{Literatur}

Aguinis, H., Beaty, J. C., Boik, R. J., \& Pierce, C. A. (2005). Effect size and power in assessing moderating effects of categorical variables using multiple regression: A 30year review. Journal of Applied Psychology, 90(1), 94-107.

Baumert, J., Blum, W., Brunner, M., Dubberke, T., Jordan, A., Klusmann, U., \& Krauss, S. (2009). Professionswissen von Lehrkräften, kognitiv aktivierender Mathematikunterricht und die Entwicklung von mathematischer Kompetenz (COACTIV): Dokumentation der Erhebungsinstrumente. Berlin: Max-Planck-Institut für Bildungsforschung.

Benjamini, Y., \& Hochberg, Y. (1995). Controlling the false discovery rate: A practical and powerful approach to multiple testing. Journal of the Royal Statistical Society. Series B (Methodological), 57(1), 289-300.

Brisson, B. M., Dicke, A.-L., Gaspard, H., Häfner, I., Flunger, B., Nagengast, B., \& Trautwein, U. (2017). Short intervention, sustained effects: Promoting students' math competence beliefs, effort, and achievement. American Educational Research Journal, 54(6), 1048-1078.

Brunner, M., Keller, U., Wenger, M., Fischbach, A., \& Lüdtke, O. (2018). Between-school variation in students' achievement, motivation, affect, and learning strategies: Results from 81 countries for planning group-randomized trials in education. Journal of Research on Educational Effectiveness, 11(3), 452-478.

Bulus, M., Dong, N., Kelcey, B., \& Spybrook, J. (2019). PowerUpR: Power analysis tools for multilevel randomized experiments. $R$ package version 1.0.3.

Collins, L. M., Schafer, J. L., \& Kam, C.-M. (2001). A comparison of inclusive and restrictive strategies in modern missing data procedures. Psychological Methods, 6(4), 330-351.

Dietrich, J., Dicke, A.-L., Kracke, B., \& Noack, P. (2015). Teacher support and its influence on students' intrinsic value and effort: Dimensional comparison effects across subjects. Learning and Instruction, 39, 45-54. 
Dong, N., Reinke, W. M., Herman, K. C., Bradshaw, C. P., \& Murray, D. W. (2016). Meaningful effect sizes, intraclass correlations, and proportions of variance explained by covariates for planning two- and three-level cluster randomized trials of social and behavioral outcomes. Evaluation Review, 40(4), 334-377.

Eccles, J. S. (2005). Subjective task value and the Eccles et al. model of achievement-related choices. In A. J. Elliot \& C. S. Dweck (Hrsg.), Handbook of competence and motivation (S. 105-121). New York, NY: The Guilford Press.

Eccles, J. S. (2007). Families, schools, and developing achievement-related motivations and engagement. In J. E. Grusec \& P. D. Hastings (Hrsg.), Handbook of socialization. Theory and research (S. 665-691). New York, NY: The Guilford Press.

Eccles, J. S., Adler, T. F., Futterman, R., Goff, S. B., Kaczala, C. M., Meece, J. L., \& Midgley, C. (1983). Expectancies, values, and academic behaviors. In J.T. Spence (Hrsg.), Achievement and achievement motives (S. 75-146). San Francisco, CA: W. H. Freeman and Company.

Enders, C. K., \& Tofighi, D. (2007). Centering predictor variables in cross-sectional multilevel models: A new look at an old issue. Psychological Methods, 12(2), 121-138.

Frenzel, A. C., Becker-Kurz, B., Pekrun, R., Goetz, T., \& Lüdtke, O. (2018). Emotion transmission in the classroom revisited: A reciprocal effects model of teacher and student enjoyment. Journal of Educational Psychology, 110(5), 628-639.

Frenzel, A. C., Goetz, T., Pekrun, R., \& Watt, H. M. G. (2010). Development of mathematics interest in adolescence: Influences of gender, family, and school context. Journal of Research on Adolescence, 20(2), 507-537.

Funder, D. C., \& Ozer, D. J. (2019). Evaluating effect size in psychological research: Sense and nonsense. Advances in Methods and Practices in Psychological Science, 2(2), 156168.

Gaspard, H., Dicke, A.-L., Flunger, B., Brisson, B. M., Häfner, I., Nagengast, B., \& 
Trautwein, U. (2015a). Fostering adolescents' value beliefs for mathematics with a relevance intervention in the classroom. Developmental Psychology, 51(9), 1226-1240.

Gaspard, H., Dicke, A.-L., Flunger, B., Schreier, B., Häfner, I., Trautwein, U., \& Nagengast, B. (2015b). More value through greater differentiation: Gender differences in value beliefs about math. Journal of Educational Psychology, 107(3), 663-677.

Gelman, A., \& Carlin, J. (2014). Beyond power calculations: Assessing Type S (sign) and Type M (magnitude) errors. Psychological Science, 9(6), 641-651.

Graham, J. W. (2003). Adding missing-data-relevant variables to FIML-based structural equation models. Structural Equation Modeling, 10(1), 80-100.

Graham, J. W. (2009). Missing data analysis: Making it work in the real world. Annual Review of Psychology, 60, 549-576.

Häfner, I., Flunger, B., Dicke, A.-L., Gaspard, H., Brisson, B. M., Nagengast, B., \& Trautwein, U. (2017). Robin Hood effects on motivation in math: Family interest moderates the effects of relevance interventions. Developmental Psychology, 53(8), $1522-1539$.

Hanushek, E. A. (2011). Valuing teachers: How much is a good teacher worth? Education Next, 11(3), 40-45.

Harackiewicz, J. M., Tibbetts, Y., Canning, E., \& Hyde, J. S. (2014). Harnessing values to promote motivation in education. In S.A. Karabenick \& T.C. Urdan (Hrsg.), Motivational interventions (Advances in Motivation and Achievement, Volume 18) (S. 71-105). Bingley: Emerald Group Publishing Limited.

Heller, K. A., \& Perleth, C. (2000). Kognitiver Fähigkeitstest für 4.-12. Klassen, Revision (KFT 4-12 R). Göttingen: Hogrefe.

Hill, C. J., Bloom, H. S., Black, A. R., \& Lipsey, M. W. (2008). Empirical benchmarks for interpreting effect sizes in research. Child Development Perspectives, 2(3), 172-177.

Hulleman, C. S., \& Harackiewicz, J. M. (2009). Promoting interest and performance in high 
school science classes. Science, 326, 1410-1412.

Joyce, K. E., \& Cartwright, N. (2020). Bridging the gap between research and practice: Predicting what will work locally. American Educational Research Journal, 57(3), 10451082.

Keller, M. M., Hoy, A. W., Goetz, T., \& Frenzel, A. C. (2016). Teacher enthusiasm: Reviewing and redefining a complex construct. Educational Psychology Review, 28(4), $743-769$.

Kunter, M., Klusmann, U., Baumert, J., Richter, D., Voss, T., \& Hachfeld, A. (2013). Professional competence of teachers: Effects on instructional quality and student development. Journal of Educational Psychology, 105(3), 805-820.

Lazarides, R., Buchholz, J., \& Rubach, C. (2018). Teacher enthusiasm and self-efficacy, student-perceived mastery goal orientation, and student motivation in mathematics classrooms. Teaching and Teacher Education, 69, 1-10.

Lazarides, R., Gaspard, H., \& Dicke, A.-L. (2019). Dynamics of classroom motivation: Teacher enthusiasm and the development of math interest and teacher support. Learning and Instruction, 60, 126-137.

Lazowski, R. A., \& Hulleman, C. S. (2016). Motivation interventions in education: A metaanalytic review. Review of Educational Research, 86(2), 602-640.

Linnenbrink, E. A., \& Pintrich, P. R. (2002). Motivation as an enabler for academic success. School Psychology Review, 31(3), 313-327.

Marsh, H. W., Lüdtke, O., Robitzsch, A., Trautwein, U., Asparouhov, T., Muthén, B., \& Nagengast, B. (2009). Doubly-latent models of school contextual effects: Integrating multilevel and structural equation approaches to control measurement and sampling error. Multivariate Behavioral Research, 44, 764-802.

Moerbeek, M. (2006). Power and money in cluster randomized trials: When is it worth measuring a covariate? Statistics in Medicine, 25(15), 2607-2617. 
Muthén, L. K., \& Muthén, B. O. (2002). How to use a Monte Carlo study to decide on sample size and determine power. Structural Equation Modeling, 9(4), 599-620.

Muthén, L. K., \& Muthén, B. O. (1998-2015). Mplus user's guide. (Seventh Ed.). Los Angeles, CA: Muthén \& Muthén.

Rakoczy, K., Klieme, E., \& Pauli, C. (2008). Die Bedeutung der wahrgenommenen Unterstützung motivationsrelevanter Bedürfnisse und des Alltagsbezugs im Mathematikunterricht für die selbstbestimmte Motivation. Zeitschrift für Pädagogische Psychologie, 22(1), 25-35.

Raudenbush, S. W., \& Liu, X. (2000). Statistical power and optimal design for multisite randomized trials. Psychological Methods, 5(2), 199-213.

Reeve, J., Jang, H., Carrell, D., Jeon, S., \& Barch, J. (2004). Enhancing students' engagement by increasing teachers' autonomy support. Motivation and Emotion, 28(2), 147-169.

Rights, J. D., \& Sterba, S. K. (2020). New recommendations on the use of R-squared differences in multilevel model comparisons. Multivariate Behavioral Research, 55(4), 568-599.

Rosenzweig, E. Q., \& Wigfield, A. (2016). STEM motivation interventions for adolescents: A promising start, but further to go. Educational Psychologist, 51(2), 146-163.

Scherrer, V., \& Preckel, F. (2019). Development of motivational variables and self-esteem during the school career: A meta-analysis of longitudinal studies. Review of Educational Research, 89(2), 211-258.

Schmidt, J. A., Kafkas, S. S., Maier, K. S., Shumow, L., \& Kackar-Cam, H. Z. (2019). Why are we learning this? Using mixed methods to understand teachers' relevance statements and how they shape middle school students' perceptions of science utility. Contemporary Educational Psychology, 57, 9-31.

Schmidt, S., Ennemoser, M., \& Krajewski, K. (2013). Deutscher Mathematiktest für neunte Klassen (DEMAT 9). Mit Ergänzungstest Konventions- und Regelwissen. Göttingen: 
Hogrefe.

Schreier, B. M., Dicke, A.-L., Gaspard, H., Häfner, I., Flunger, B., Lüdtke, O., Nagengast, B., \& Trautwein, U. (2014). Der Wert der Mathematik im Klassenzimmer - Die Bedeutung relevanzbezogener Unterrichtsmerkmale für die Wertüberzeugungen der Schülerinnen und Schüler. Zeitschrift für Erziehungswissenschaft, 17, 225-255.

Snijders, T. A. B. (2005). Power and sample size in multilevel linear models. In B. S. Everitt \& D. C. Howell (Hrsg.), Encyclopedia of statistics in behavioral science (S. 1570-1573). Chichester, UK: Wiley.

Spybrook, J., Kelcey, B., \& Dong, N. (2016). Power for detecting treatment by moderator effects in two- and three-level cluster randomized trials. Journal of Educational and Behavioral Statistics, 41(6), 605-627.

Stallasch, S. E., Lüdtke, O., Artelt, C., \& Brunner, M. (2021). Multilevel design parameters to plan cluster-randomized intervention studies on student achievement in elementary and secondary school. Journal of Research on Educational Effectiveness, Advance online publication.

Walton, G. M., \& Brady, S. T. (2020). The social-belonging intervention. In G. M. Walton \& A. J. Crum (Hrsg.), Handbook of wise interventions: How social-psychological insights can help solves problems. New York, NY: Guildford Press.

Walton, G. M., \& Yeager, D. S. (2020). Seed and soil: Psychological affordances in contexts explain where wise interventions succeed or fail. Current Directions in Psychological Science, Advance online publication.

Watt, H. M. G. (2004). Development of adolescents's self-perceptions, values, and task perceptions according to gender and domain in 7th-through 11th-grade Australian students. Child Development, 75(5), 1556-1574.

Wentzel, K. R. (2009). Students' relationships with teachers as motivational contexts. In K. R. Wentzel \& A. Wigfield (Eds.), Handbook of motivation at school (pp. 301-322). New 
York, NY: Routledge.

Wentzel, K. R., Battle, A., Russell, S. L., \& Looney, L. B. (2010). Social supports from teachers and peers as predictors of academic and social motivation. Contemporary Educational Psychology, 35(3), 193-202.Wentzel, K. R., \& Wigfield, A. (Hrsg.) (2009). Handbook of motivation at school. New York, NY: Routledge.

Yeager, D. S., Hanselman, P., Walton, G. M., Murray, J. S., Crosnoe, R., Muller, C., Tipton, E., Schneider, B., Hulleman, C. S., Hinojosa, C. P., Paunesku, D., Romero, C., Flint, K., Roberts, A., Trott, J., Iachan, R., Buontempo, J., Yang, S. M., Carvalho, C. M., Hahn, P. R., Gopalan, M., Mhatre, P., Ferguson, R., Duckworth, A. L., \& Dweck, C. S. (2019). A national experiment reveals where a growth mindset improves achievement. Nature, 573, 364-369.

Zhang, Q. (2013). Assessing the effects of instructor enthusiasm on classroom engagement, learning goal orientation, and academic self-efficacy. Communication Teacher, 28(1), 44-56. 